\title{
Fatigue Load Sensitivity Based Optimal Active Power Dispatch For Wind Farms
}

Zhao, Haoran; Wu, Qiuwei; Huang, Shaojun; Shahidehpour, Mohammad ; Guo, Qinglai; Sun, Hongbin

Published in:

IEEE Transactions on Sustainable Energy

Link to article, DOI:

10.1109/TSTE.2017.2673122

Publication date:

2017

Document Version

Peer reviewed version

Link back to DTU Orbit

Citation (APA):

Zhao, H., Wu, Q., Huang, S., Shahidehpour, M., Guo, Q., \& Sun, H. (2017). Fatigue Load Sensitivity Based Optimal Active Power Dispatch For Wind Farms. IEEE Transactions on Sustainable Energy, 8(3), 1247-1259. https://doi.org/10.1109/TSTE.2017.2673122

\section{General rights}

Copyright and moral rights for the publications made accessible in the public portal are retained by the authors and/or other copyright owners and it is a condition of accessing publications that users recognise and abide by the legal requirements associated with these rights.

- Users may download and print one copy of any publication from the public portal for the purpose of private study or research.

- You may not further distribute the material or use it for any profit-making activity or commercial gain

- You may freely distribute the URL identifying the publication in the public portal 


\title{
Fatigue Load Sensitivity Based Optimal Active Power Dispatch For Wind Farms
}

\author{
Haoran Zhao, Qiuwei Wu, Shaojun Huang, Mohammad Shahidehpour, Qinglai Guo and Hongbin Sun
}

\begin{abstract}
This paper proposes an optimal active power dispatch algorithm for wind farms based on Wind Turbine (WT) load sensitivity. The control objectives include tracking power references from the system operator and minimizing fatigue loads experienced by WTs. The sensitivity of WT fatigue loads to power references is defined which simplifies the formulation of the optimal power dispatch problem. Since the sensitivity value is calculated at the local WT controller, the computation burden of the central wind farm controller is largely reduced. Moreover, explicit analytical equations of the fatigue load sensitivity are derived, which significantly improves the computation efficiency of the local WT controller. The proposed algorithm can be implemented in different active power control schemes. Case studies were conducted with a wind farm under balance control for both low and high wind conditions. By comparing the rainflow cycles and Damage Equivalent Load (DEL), the efficacy of the proposed algorithm is verified.
\end{abstract}

Index Terms-Fatigue load, load sensitivity, optimal active power dispatch, wind farm, wind turbine.

\section{INTRODUCTION}

$\mathbf{N}$ OWADAYS, wind power is the fastest growing Renewable Energy Resource (RES). According to the report of the European Wind Energy Association (EWEA), the newly installed wind power capacity of EU in 2014 is $12.8 \mathrm{GW}$ [1]. By the end of 2015, the cumulative capacity of wind power had reached $141.6 \mathrm{GW}$, which can meet around $11.4 \%$ of Europe's electricity demand.

With the increasing penetration level, variability and uncertainty of wind power have brought new technical challenges to power system operation [2]-[4]. The technical requirements for wind power integration are more stringent [5]. For the active power, different control requirements are specified, such as balance control, delta limitation, ramp rate limitation, etc. [6]. In other words, a wind farm shall be capable of tracking the power reference from the system operator.

With fast development of power electronics, controllability of modern Wind Turbines (WTs) has been largely improved.

H. Zhao and S. Huang are with Center for Electric Power and Energy (CEE), Department of Electrical Engineering, Technical University of Denmark (DTU), Kgs. Lyngby, 2800 Denmark (e-mail: hzhao@elektro.dtu.dk; shuang@elektro.dtu.dk).

Q. Wu is with Center for Electric and Energy (CEE), Department of Electrical Engineering, Technical University of Denmark (DTU), Kgs. Lyngby, 2800 Denmark, and School of Electrical Engineering, Shandong University, China (e-mail: qw@elektro.dtu.dk).

M. Shahidehpour is with Electrical and Computer Engineering Department, Illinois Institute of Technology (IIT), Chicago, IL 60616, USA (e-mail: ms@iit.edu).

Q. Guo and H. Sun are with Department of Electrical Engineering, Tsinghua University, Beijing, 100084 China (e-mail: guoqinglai@tsinghua.edu.cn; shb@mail.tsinghua.edu.cn).
When required wind farm power is less than the maximum available power, WTs will limit power production and operate in the derated mode. In order to achieve specific control objectives, it is very important to optimally dispatch power references to individual WTs.

The initial dispatch schemes for wind farms only focus on the power reference tracking. The power reference is proportionally distributed to individual WTs according to either the available power [7] or the actual output power [8]. Fatigue loads (load for short hereinafter) experienced by WTs, which has a significant impact on the lifetime of WTs, are not considered.

In recent studies, several multi-objective dispatch algorithms have been proposed [9]-[18]. The studies of [9]-[11] focus on the multi-objective control at the WT level. On top of providing the desired power production, mechanical loads are minimized by coordinating the pitch angle and electrical torque. For the wind farm level control, as long as the power requirement specified by the system operator is met, loads can be minimized by coordination among WTs [19]. The optimal control problem is either solved in a centralized manner [12][14] or a distributed manner [15]-[18]. For the former case, the wind farm model is formulated as a coupled, constrained Multiple Input and Multiple Output (MIMO) system whose order drastically grows with increasing the number of WTs. Since a modern large-scale wind farm may consist of several hundred WTs, the computation burden of the centralized control scheme is quite heavy and it cannot be used for realtime application. For the latter case, the distributed control scheme can efficiently reduce the computation burden. However, in order to guarantee the optimality, the iteration among WTs is required which implies high sampling communication required.

In this paper, a load sensitivity based optimal active power dispatch algorithm is proposed for wind farms to overcome the aforementioned problems. It minimizes WT loads while tracking the wind farm power reference. The proposed approach includes the following two aspects. Firstly, the sensitivity of WT fatigue loads to power references is defined and introduced as the signal exchanged between the central and local controllers. Secondly, explicit expression of the WT load sensitivities are derived.

The main contribution is that the proposed scheme enables efficient implementation of the optimal wind farm control, especially for large-scale wind farms. The proposed scheme is solved in the centralized manner. Compared with the other central optimal wind farm control schemes, the formulation of the optimal dispatch algorithm is simplified. Most of 
the computation task is undertaken by the local controller and solved in parallel. The explicit expressions of the load sensitivities can reduce the online computation burden of the local controller. Moreover, the optimal control problem is formulated as a standard Quadratic Programming (QP) problem and can be efficiently solved. Compared with the distributed control scheme, the global optimum of the QP problem can be obtained without additional iterations.

The paper is organized as follows. Section II describes the structure of the wind farm control based on the load sensitivity. The modeling and operation of a power-controlled WT are introduced in Section III and Section IV, respectively. The explicit equations of load sensitivities are derived in Section V. Section VI explains the formulation of the optimal dispatch algorithm. Case studies are presented and discussed in Section VII, followed by conclusions.

\section{LOAD SENSITIVITy BASED Wind FARM CONTROL STRUCTURE}

The proposed wind farm control structure is shown in Fig. 1. According to the requirement of the system operator and the available power $P_{\mathrm{avi}}^{\mathrm{wfc}}$, the active power control scheme of the wind farm is decided. The power reference of the wind farm $P_{\mathrm{ref}}^{\mathrm{wfc}}$ is calculated and delivered to the wind farm controller. In the optimal wind farm control schemes of the previous studies, the measurements of individual WTs are sent directly to the central wind farm controller and the optimization problem is formulated based on a complicated wind farm model.



Fig. 1. Wind farm control structure.

In the proposed wind farm control, the load sensitivity $\frac{\partial L}{\partial P^{\mathrm{w} t}}$ and the power reference constraints of individual WTs $\left(\left[P_{\mathrm{min}}^{\mathrm{w}}, P_{\mathrm{max}}^{\mathrm{wt}}\right]\right)$ are used for the formulation of the optimal dispatch problem. They are calculated locally and sent to the wind farm controller. The details of calculating $\frac{\partial L}{\partial P_{\text {ref }}^{\mathrm{we}}}$ and constraints $\left(\left[P_{\mathrm{min}}^{\mathrm{wt}}, P_{\max }^{\mathrm{wt}}\right]\right)$ are described in Section IV and Section $\mathrm{V}$, respectively. By solving the optimization problem, the power references of individual WTs $P_{\text {ref }}^{\text {wt }}$ are updated for each interval of wind farm control. During the control interval, based on $P_{\text {ref }}^{\mathrm{wt}}$, a torque reference $T_{\mathrm{g}_{\mathrm{r}} \text { ref }}$ for the local torque control and a pitch angle reference $\theta_{\text {ref }}$ for the pitch actuator are generated. The control interval of the local controller is smaller.

\section{Modeling of A Power-Controlled WT}

In the wind farm control, the role of individual WTs is an actuator, which operates in a derated condition and tracks $P_{\text {ref }}^{\mathrm{wt}}$. The power-controlled WT model developed by National Renewable Energy Laboratory (NREL) is used to represent a variable speed pitch-controlled WT [20], [21]. The model structure is shown in Fig. 2.

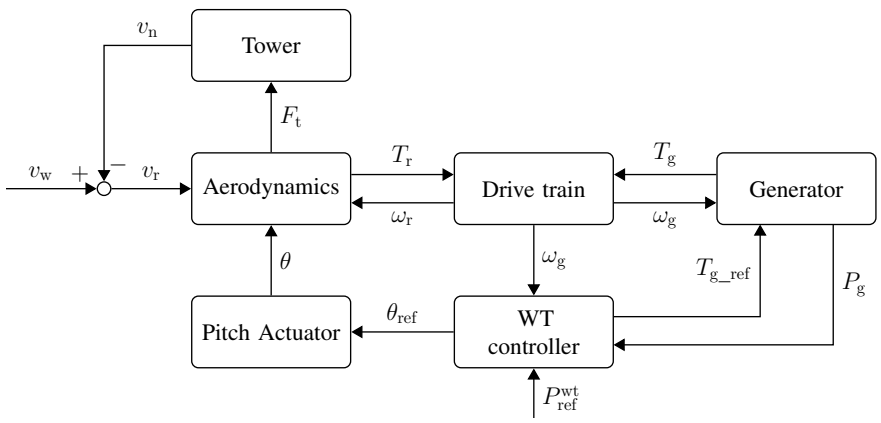

Fig. 2. Power-controlled WT model [21].

Normally, the sampling time of the wind farm controller $t_{\mathrm{s}}$ is in seconds [17]. Therefore, the fast dynamics in the generator and pitch actuator can be ignored [12]. Moreover, the oscillations in the shaft torsion and tower nodding are disregarded to reduce the model complexity. In this study, a simplified nonlinear WT model with its local control is introduced in the wind farm control, which is used to calculate the load sensitivities. Its controller and operation are described in Section IV.

\section{A. Aerodynamics}

The aerodynamic torque $T_{\mathrm{a}}$ and thrust force $F_{\mathrm{t}}$ are the main sources of nonlinearities [22], calculated by,

$$
\begin{aligned}
T_{\mathrm{a}} & =\frac{0.5 \pi \rho R^{2} v_{\mathrm{r}}^{3} C_{\mathrm{p}}(\lambda, \theta)}{\omega_{\mathrm{r}}}, \\
F_{\mathrm{t}} & =0.5 \pi \rho R^{2} v_{\mathrm{r}}^{2} C_{\mathrm{t}}(\lambda, \theta),
\end{aligned}
$$

where $C_{\mathrm{p}}$ and $C_{\mathrm{t}}$ are the power coefficient and thrust coefficient, respectively, $R$ is the length of the blade, $\rho$ is the air density, $v_{\mathrm{r}}$ is the effective wind speed on the rotor, and $\lambda$ is the tip speed ratio, defined by $\lambda \triangleq \frac{\omega_{\mathrm{r}} R}{v_{\mathrm{r}}}$.

\section{B. Drive Train}

The drive train is considered to be rigidly coupled and the single-mass model is used in this study, where the rotor mass $J_{\mathrm{r}}$ and generator mass $J_{\mathrm{g}}$ are merged into one equivalent mass $J_{\mathrm{t}}$ [23], expressed by,

$$
J_{\mathrm{t}}=J_{\mathrm{r}}+\eta_{\mathrm{g}}^{2} J_{\mathrm{g}} .
$$

According to the low-shaft motion equation,

$$
\begin{gathered}
\dot{\omega}_{\mathrm{r}}=\frac{1}{J_{\mathrm{t}}}\left(T_{\mathrm{a}}-\eta_{\mathrm{g}} T_{\mathrm{g}}\right), \\
\omega_{\mathrm{g}}=\eta_{\mathrm{g}} \omega_{\mathrm{r}},
\end{gathered}
$$

where $\omega_{\mathrm{r}}$ and $\omega_{\mathrm{g}}$ are the rotor and generator speeds, respectively, and $\eta_{\mathrm{g}}$ is the gear box ratio. 


\section{Generator}

In the torque control loop, the vector control is used to ensure a fast (in milliseconds) and accurate response [24]. Since the dynamic is neglected, the generator torque $T_{\mathrm{g}}$ is approximately equal to its reference, i.e.,

$$
T_{\mathrm{g}} \approx T_{\mathrm{g} \_ \text {ref }} .
$$

\section{Tower}

The tower dynamics is not included in the simplified WT model. According to [14], [25], it is assumed the fore-aft bending moment at the tower base $M_{\mathrm{t}}$ can be approximately derived by,

$$
M_{\mathrm{t}} \approx H \cdot F_{\mathrm{t}}
$$

where $H$ indicates the tower height.

\section{OPERATION AND CONTROL OF WTS IN A WIND FARM}

Conventionally, WTs are controlled to track its Optimal Regimes Characteristic (ORC), as shown in Fig. 3. The polyhedron marked in the figure shows the feasible operation area where the power coefficient $C_{\mathrm{p}}>0$. Define the rated wind speed as $v_{\text {rated }}$ and the rated power as $P_{\text {rated }}^{\text {wt }}$ When $v_{\mathrm{w}} \leq v_{\text {rated }}$, the pitch control is deactivated $(\theta=0)$ and $T_{\mathrm{g}}$ is regulated to track the optimal rotor speed. The maximum available wind power is extracted $\left(P_{\text {ref }}^{\mathrm{wt}}=P_{\text {avi }}^{\mathrm{wt}} \leq P_{\text {rated }}^{\mathrm{wt}}\right)$. When $v_{\mathrm{w}}>v_{\text {rated }}, T_{\mathrm{g}}$ is kept at its rated value and the pitch control is activated to prevent the generator speed $\omega_{\mathrm{g}}$ from over-speeding. The captured wind power is limited to the rated value $\left(P_{\text {ref }}^{\mathrm{wt}}=P_{\text {rated }}^{\mathrm{wt}}\right)$.

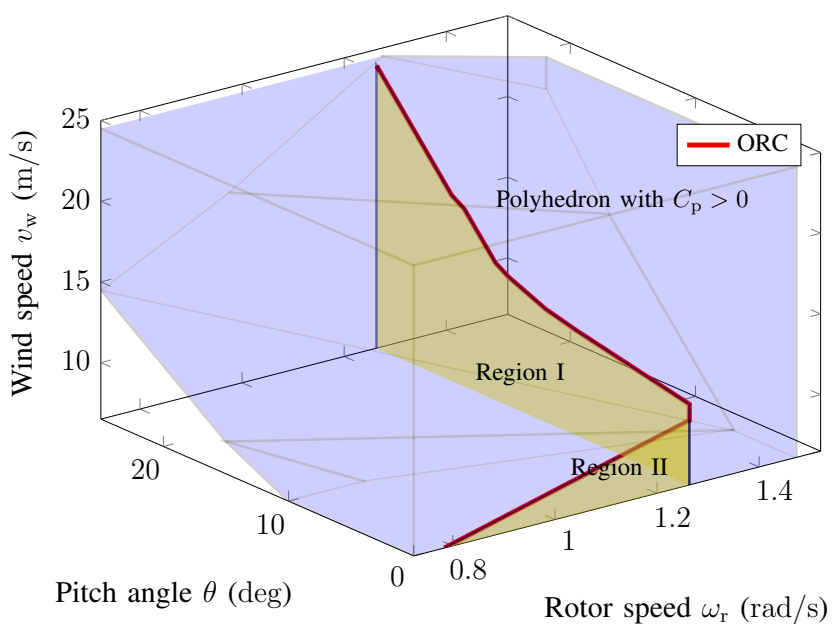

Fig. 3. Operation regions of WT [16].

In [20], the WT operates in the power maximization mode. In this study, the WT operates in the derated mode, i.e., $P_{\text {ref }}^{\mathrm{wt}}<$ $P_{\mathrm{avi}}^{\mathrm{wt}}$. Different from the region definition in [20], the operation area is below the ORC and can be divided into two regions according to the status of the pitch control, as shown in Fig. 3. In order to follow $P_{\text {ref }}^{\mathrm{wt}}$, the pitch angle reference $\theta_{\text {ref }}$ and generator torque reference $T_{\text {g_ref }}$ are computed by the WT controller and then sent to the pitch actuator and generator, respectively.

\section{A. Region I}

The pitch control is activated. The captured power is controlled by regulating $\theta_{\text {ref }}$ and $T_{\mathrm{g} \_ \text {ref }}$. The measured generator speed $\omega_{\mathrm{g}}$ is filtered by a low-pass filter and the filtered speed $\omega_{\mathrm{f}}$ is derived by,

$$
\omega_{\mathrm{f}}=\frac{1}{1+s \tau_{\mathrm{f}}} \omega_{\mathrm{g}},
$$

where $\tau_{\mathrm{f}}$ is the time constant of the filter.

According to the deviation of $\omega_{\mathrm{f}}$ from its rated value $\omega_{\mathrm{g} \_ \text {rate }}$, $\theta_{\text {ref }}$ is obtained by the gain-scheduled PI controller,

$$
\theta_{\text {ref }}=\frac{K_{\mathrm{p}}}{K_{\mathrm{c}}}\left(\omega_{\mathrm{f}}-\omega_{\mathrm{g} \_ \text {rate }}\right)+\frac{K_{\mathrm{i}}}{K_{\mathrm{c}}} \frac{\omega_{\mathrm{f}}-\omega_{\mathrm{g} \_ \text {rate }}}{s},
$$

where $K_{\mathrm{p}}$ and $K_{\mathrm{i}}$ are proportional and integral gains of the PI controller, respectively, and $K_{\mathrm{c}}$ is the correction factor. In [21], $K_{\mathrm{c}}$ is a function of $\theta$, i.e., $K_{\mathrm{c}}=K_{0}+K_{\theta} \theta$, where $K_{0}$ and $K_{\theta}$ are the constants.

$T_{\mathrm{g} \_ \text {ref }}$ is calculated by,

$$
T_{\mathrm{g} \_ \text {ref }}=\frac{P_{\text {ref }}^{\mathrm{wt}}}{\omega_{\mathrm{f}}} .
$$

\section{B. Region II}

The pitch control is deactivated, i.e., $\theta_{\text {ref }}=0$. The captured power is only controlled by regulating $T_{\mathrm{g} \_ \text {ref }}$, which is also calculated by (10).

\section{Sensitivity Calculation of WT LoAd}

The fatigue loads of WTs can be divided into aerodynamic and gravity loads (external), and structural loads (internal) [26]. In this paper, the fatigue loads mainly focus on the loads of the drive train due to the torsion of the shaft and the loads of the tower structure due to the tower deflection. Compared with static loads, the dynamic stress causing structural damage of WTs is a much bigger issue. By damping the fluctuations of low-speed shaft torque $T_{\mathrm{s}}$ and thrust force $F_{\mathrm{t}}$, the related fatigue loads can be reduced. Accordingly, for the drive train loads, the load sensitivity $\frac{\partial L}{\partial P_{\mathrm{ref}}^{\mathrm{wt}}}$ can be represented by $\frac{\partial T_{\mathrm{s}}}{\partial P_{\mathrm{ref}}^{\mathrm{wt}}}$. For the tower structure loads, $\frac{\partial L}{\partial P_{\mathrm{ref}}^{\mathrm{wt}}}$ can be represented by $\frac{\partial F_{\mathrm{t}}}{\partial P_{\mathrm{ref}}^{\mathrm{ww}}}$. If both drive train and tower structure loads are considered, $\frac{\partial L}{\partial P_{\mathrm{wef}}^{\mathrm{wt}}}$ can be represented by the combination of $\frac{\partial T_{\mathrm{s}}}{\partial P_{\mathrm{ref}}^{\mathrm{wt}}}$ and $\frac{\partial F_{\mathrm{t}}}{\partial P_{\mathrm{ref}}^{\mathrm{wt}}}$.

\section{A. Incremental State Space Model}

Suppose the time of the operating point is $t_{0}$. The wind speed $v_{\mathrm{r}}$ is a variable which can either be measured or estimated [27]. In this study, $v_{\mathrm{r}}$ is estimated. The value at $t=t_{0}$ is $v_{\mathrm{r} 0}$ and assumed to be constant for the short control period. The measured power production, generator speed, filtered speed and pitch angle at $t=t_{0}$ are defined as $P_{\mathrm{g} 0}, \omega_{\mathrm{g} 0}, \omega_{\mathrm{f} 0}$, and $\theta_{0}$, respectively. According to (1) and (10), the aerodynamic torque and generator torque at $t=t_{0}$ can be calculated and are defined as $T_{\mathrm{g} 0}$ and $T_{\mathrm{a} 0}$, respectively.

Based on linearization of the simplified nonlinear WT model at the operating point, the incremental state space model for Region I is derived by the following steps. Then, the model 
for Region II can be derived by neglecting the term related to the pitch angle. The sign $\Delta$ indicates the increment of a variable.

Step 1: Derivative equations of $\Delta \omega_{\mathrm{g}}, \Delta \omega_{\mathrm{f}}$ and $\Delta \beta$.

Based on (4) and (5),

$$
\Delta \dot{\omega}_{\mathrm{g}}=\frac{\eta_{\mathrm{g}}}{J_{\mathrm{t}}}\left(\Delta T_{\mathrm{a}}-\eta_{\mathrm{g}} \Delta T_{\mathrm{g}}\right)+\frac{\eta_{\mathrm{g}}}{J_{\mathrm{t}}}\left(T_{\mathrm{a} 0}-\eta_{\mathrm{g}} T_{\mathrm{g} 0}\right) .
$$

Based on (8),

$$
\Delta \dot{\omega}_{\mathrm{f}}=-\frac{1}{\tau_{\mathrm{f}}} \Delta \omega_{\mathrm{f}}+\frac{1}{\tau_{\mathrm{f}}} \Delta \omega_{\mathrm{g}} .
$$

By defining $\beta \triangleq K_{\mathrm{c}} \theta=K_{0} \theta+K_{\theta} \theta^{2}$, (8) is transformed into,

$$
\beta=K_{\mathrm{p}}\left(\omega_{\mathrm{f}}-\omega_{\mathrm{g} \_ \text {rate }}\right)+K_{\mathrm{i}} \frac{\omega_{\mathrm{f}}-\omega_{\mathrm{g} \_ \text {rate }}}{s} .
$$

Based on (12) and (13),

$$
\begin{aligned}
\dot{\Delta \beta} & =K_{\mathrm{p}} \Delta \dot{\omega_{\mathrm{f}}}+K_{\mathrm{i}}\left(\omega_{\mathrm{f} 0}+\Delta \omega_{\mathrm{f}}-\omega_{\mathrm{g}_{-} \text {rated }}\right) \\
& =\frac{K_{\mathrm{p}}}{\tau_{\mathrm{f}}} \Delta \omega_{\mathrm{g}}+\left(-\frac{K_{\mathrm{p}}}{\tau_{\mathrm{f}}}+K_{\mathrm{i}}\right) \Delta \omega_{\mathrm{f}}+K_{\mathrm{i}}\left(\omega_{\mathrm{f} 0}-\omega_{\mathrm{g} \_ \text {rated }}\right) .
\end{aligned}
$$

Step 2: Calculation of $\Delta T_{\mathrm{a}}$ and $\Delta T_{\mathrm{g}}$.

Equation (10) is nonlinear. It could be approximately calculated around the operating point according to the Taylor series,

$$
\Delta T_{\mathrm{g}} \approx-\frac{P_{\mathrm{g} 0}}{\omega_{\mathrm{f} 0}^{2}} \Delta \omega_{\mathrm{f}}+\frac{1}{\omega_{\mathrm{f} 0}} \Delta P_{\mathrm{ref}}^{\mathrm{wt}} .
$$

Similarly, according to (1), $\Delta T_{\mathrm{a}}$ can be calculated by,

$$
\left.\Delta T_{\mathrm{a}} \approx \frac{\partial T_{\mathrm{a}}}{\partial \omega_{\mathrm{g}}}\right|_{\left(\omega_{\mathrm{g} 0}, v_{\mathrm{r} 0}, \theta_{0}\right)} \Delta \omega_{\mathrm{g}}+\left.\frac{\partial T_{\mathrm{a}}}{\partial \beta}\right|_{\left(\omega_{\mathrm{g} 0}, v_{\mathrm{r} 0}, \theta_{0}\right)} \Delta \beta .
$$

In the following, $\frac{\partial T_{\mathrm{a}}}{\partial \omega_{\mathrm{g}}}$ and $\frac{\partial T_{\mathrm{a}}}{\partial \beta}$ are used for $\left.\frac{\partial T_{\mathrm{a}}}{\partial \omega_{\mathrm{g}}}\right|_{\left(\omega_{\mathrm{g} 0}, v_{\mathrm{r} 0}, \theta_{0}\right)}$ and $\left.\frac{\partial T_{\mathrm{a}}}{\partial \beta}\right|_{\left(\omega_{\mathrm{g} 0}, v_{\mathrm{r} 0}, \theta_{0}\right)}$, respectively.

Step 3: Calculation of $\frac{\partial T_{\mathrm{a}}}{\partial \omega_{\mathrm{g}}}$ and $\frac{\partial T_{\mathrm{a}}}{\partial \beta}$.

By defining $P_{0} \triangleq 0.5 \pi \rho R^{2} v_{\mathrm{r} 0}^{3}$, the partial derivatives of $T_{\mathrm{a}}$ are derived according to (1),

$$
\begin{gathered}
\frac{\partial T_{\mathrm{a}}}{\partial \omega_{\mathrm{g}}}=-\frac{\eta_{\mathrm{g}} P_{0} C_{\mathrm{p}}\left(\omega_{\mathrm{g} 0}, v_{\mathrm{r} 0}, \theta_{0}\right)}{\omega_{\mathrm{g} 0}^{2}}+\frac{\eta_{\mathrm{g}} P_{0}}{\omega_{\mathrm{g} 0}} \frac{\partial C_{\mathrm{p}}\left(\omega_{\mathrm{g}}, v_{\mathrm{r} 0}, \theta_{0}\right)}{\partial \omega_{\mathrm{g}}} \\
\frac{\partial T_{\mathrm{a}}}{\partial \theta}=\frac{\eta_{\mathrm{g}} P_{0}}{\omega_{\mathrm{g} 0}} \frac{\partial C_{\mathrm{p}}\left(\omega_{\mathrm{g} 0}, v_{\mathrm{r} 0}, \theta\right)}{\partial \theta}
\end{gathered}
$$

Accordingly,

$$
\frac{\partial T_{\mathrm{a}}}{\partial \beta}=\frac{\partial T_{\mathrm{a}}}{\partial \theta} \frac{\partial \theta}{\partial \beta}=\frac{\partial T_{\mathrm{a}}}{\partial \theta} \frac{1}{K_{0}+2 K_{\theta} \theta_{0}} .
$$

Step 4: Calculation of $\frac{\partial C_{\mathrm{p}}}{\partial \omega_{\mathrm{g}}}$ and $\frac{\partial C_{\mathrm{p}}}{\partial \theta}$.

The function $C_{\mathrm{p}}\left(\omega_{\mathrm{g}}, v_{\mathrm{r}}, \theta\right)$ is nonlinear. Normally, it can be expressed in two formats. For the first format, $C_{\mathrm{p}}$ is described by an empirical formula [28], e.g.,

$$
\begin{gathered}
C_{\mathrm{p}}\left(\omega_{\mathrm{g}}, v_{\mathrm{r}}, \theta\right)=0.22\left(\frac{116}{\lambda_{i}}-0.4 \theta-5\right) e^{\frac{12.5}{\lambda_{i}}}, \\
\frac{1}{\lambda_{i}}=\frac{1}{\lambda+0.08 \theta}-\frac{0.035}{\theta^{3}+1}, \lambda=\frac{\eta_{\mathrm{g}} \omega_{\mathrm{g}} R}{v_{\mathrm{r}}} .
\end{gathered}
$$

For the second format, $C_{\mathrm{p}}$ is described in a lookup table derived from the geometry of the blades with inputs $\lambda$ and $\theta$ [21], as shown in Fig. 4. The differences of $\lambda$ and $\theta$ of neighbouring cells are $\Delta \lambda_{\text {tab }}$ and $\Delta \theta_{\text {tab }}$, respectively. In this study, the second format is used. The corresponding data for this study can be accessed in the wind turbine model of SimWindFarm-a publicly available Simulink toolbox for dynamic wind farm modeling, simulation, and control [21]. The plot of $C_{\mathrm{p}}(\lambda, \theta)$ based on the lookup table is shown in Fig. 5.

\begin{tabular}{c|l|l|l|l}
\hline$\theta$ & $\lambda_{\min }$ & $\lambda_{\min }+\Delta \lambda_{\text {tab }}$ & $\cdots$ & $\lambda_{\max }$ \\
\hline$\theta_{\min }$ & & & & \\
\hline$\theta_{\min }+\Delta \theta_{\mathrm{tab}}$ & & & & \\
\hline$\vdots$ & & & & \\
\hline$\theta_{\max }$ & & & & \\
\hline
\end{tabular}

Fig. 4. Lookup table format of $C_{\mathrm{p}}(\lambda, \theta)$.

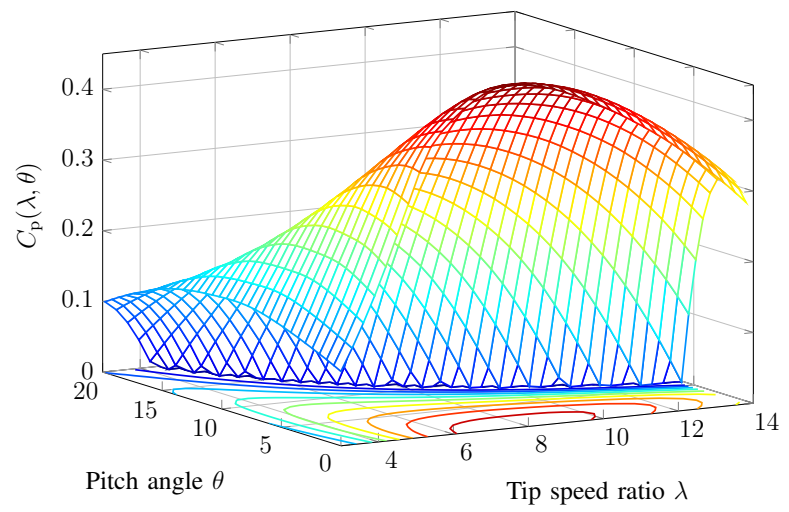

Fig. 5. $C_{\mathrm{p}}(\lambda, \theta)$ based on the data from SimWindFarm [21].

According to $v_{\mathrm{r} 0}, \omega_{\mathrm{g} 0}$, and $\theta_{0}$, the power coefficient at the operating point $C_{\mathrm{p} 0}$ can be obtained based on the lookup table, i.e., $C_{\mathrm{p} 0}=C_{\mathrm{p}(n, m)}$, where $n$ and $m$ are the corresponding row and column indices, respectively. $\frac{\partial C_{\mathrm{p}}}{\partial \omega_{\mathrm{g}}}$ and $\frac{\partial C_{\mathrm{p}}}{\partial \theta}$ can be calculated by,

$$
\begin{gathered}
\frac{\partial C_{\mathrm{p}}}{\partial \omega_{\mathrm{g}}}=\frac{R \eta_{\mathrm{g}}}{v_{\mathrm{r} 0}} \frac{\partial C_{\mathrm{p}}}{\partial \lambda}, \frac{\partial C_{\mathrm{p}}}{\partial \lambda} \approx \frac{C_{\mathrm{p}(n, m+1)}-C_{\mathrm{p} 0}}{\Delta \lambda_{\mathrm{tab}}}, \\
\frac{\partial C_{\mathrm{p}}}{\partial \theta} \approx \frac{C_{\mathrm{p}(n+1, m)}-C_{\mathrm{p} 0}}{\Delta \theta_{\mathrm{tab}}} .
\end{gathered}
$$

\section{Step 5: Formulation of the state space model.}

According to (11)-(22), the continuous state space model for Region I is formulated as,

$$
\dot{x}=\boldsymbol{A}_{\mathbf{I}} x+\boldsymbol{B}_{\mathbf{I}} \Delta P_{\mathrm{ref}}^{\mathrm{wt}}+\boldsymbol{E}_{\mathbf{I}},
$$

with

$$
\begin{aligned}
& x=\left[\Delta \omega_{\mathrm{g}}, \Delta \beta, \Delta \omega_{\mathrm{f}}\right]^{\prime}, \\
& \boldsymbol{A}_{\mathrm{I}}=\left[\begin{array}{ccc}
\frac{\eta_{\mathrm{g}}}{J_{\mathrm{t}}} \frac{\partial T_{\mathrm{a}}}{\partial \omega_{\mathrm{g}}} & \frac{\eta_{\mathrm{g}}}{J_{\mathrm{t}}} \frac{\partial T_{\mathrm{a}}}{\partial \beta} & \frac{\eta_{\mathrm{g}}^{2} P_{\mathrm{g} 0}}{J_{\mathrm{t}} \omega_{\mathrm{f} 0}^{2}} \\
\frac{K_{\mathrm{p}}}{\tau_{\mathrm{f}}} & 0 & -\frac{K_{\mathrm{p}}}{\tau_{\mathrm{f}}}+K_{\mathrm{i}} \\
\frac{1}{\tau_{\mathrm{f}}} & 0 & -\frac{1}{\tau_{\mathrm{f}}}
\end{array}\right],
\end{aligned}
$$




$$
\boldsymbol{B}_{\mathbf{I}}=\left[\begin{array}{c}
-\frac{\eta_{\mathrm{g}}^{2}}{J_{\mathrm{t}} \omega_{\mathrm{f} 0}} \\
0 \\
0
\end{array}\right], \quad \boldsymbol{E}_{\mathbf{I}}=\left[\begin{array}{c}
\frac{\eta_{\mathrm{g}}}{J_{\mathrm{t}}}\left(T_{\mathrm{a} 0}-\eta_{\mathrm{g}} T_{\mathrm{g} 0}\right) \\
K_{\mathrm{i}}\left(\omega_{\mathrm{f} 0}-\omega_{\mathrm{g} \_ \text {rated }}\right) \\
0
\end{array}\right]
$$

For Region II, the terms related to the pitch angle is neglected,

$$
\begin{aligned}
& x=\left[\Delta \omega_{\mathrm{g}}, \Delta \omega_{\mathrm{f}}\right]^{\prime}, \\
& \boldsymbol{A}_{\mathbf{I I}}=\left[\begin{array}{cc}
\frac{\eta_{\mathrm{g}}}{J_{\mathrm{t}}} \frac{\partial T_{\mathrm{a}}}{\partial \omega_{\mathrm{g}}} & \frac{\eta_{\mathrm{g}}^{2} P_{\mathrm{g} 0}}{J_{\mathrm{t}} \omega_{\mathrm{f} 0}^{2}} \\
\frac{1}{\tau_{\mathrm{f}}} & -\frac{1}{\tau_{\mathrm{f}}}
\end{array}\right], \boldsymbol{B}_{\mathbf{I I}}=\left[\begin{array}{c}
-\frac{\eta_{\mathrm{g}}^{2}}{J_{\mathrm{t}} \omega_{\mathrm{f} 0}} \\
0
\end{array}\right], \\
& \boldsymbol{E}_{\mathbf{I I}}=\left[\begin{array}{c}
\frac{\eta_{\mathrm{g}}}{J_{\mathrm{t}}}\left(T_{\mathrm{a} 0}-\eta_{\mathrm{g}} T_{\mathrm{g} 0}\right) \\
0
\end{array}\right] .
\end{aligned}
$$

\section{Step 5: Discretization.}

The continuous state space model (23) is discretized with the sampling period $t_{\mathrm{s}}$.

For Region I, the discrete state space model is,

$$
x(k+1)=\boldsymbol{A}_{\mathbf{I}}^{\mathbf{d}} x(k)+\boldsymbol{B}_{\mathbf{I}}^{\mathrm{d}} \Delta P_{\mathrm{ref}}^{\mathrm{wt}}+\boldsymbol{E}_{\mathbf{I}}^{\mathbf{d}},
$$

with

$$
\boldsymbol{A}_{\mathbf{I}}^{\mathbf{d}}=e^{\boldsymbol{A}_{\mathbf{I}} t_{\mathrm{s}}}, \boldsymbol{B}_{\mathbf{I}}^{\mathbf{d}}=\int_{0}^{t_{\mathrm{s}}}\left(e^{\boldsymbol{A}_{\mathbf{I}} t} \boldsymbol{B}_{\mathbf{I}}\right) d t, \boldsymbol{E}_{\mathbf{I}}^{\mathbf{d}}=\int_{0}^{t_{\mathrm{s}}}\left(e^{\boldsymbol{A}_{\mathbf{I}} t} \boldsymbol{E}_{\mathbf{I}}\right) d t
$$

where $k$ is the step index.

For Region II, the discrete state space model is,

$$
x(k+1)=\boldsymbol{A}_{\mathbf{I I}}^{\mathbf{d}} x(k)+\boldsymbol{B}_{\mathbf{I I}}^{\mathbf{d}} \Delta P_{\mathrm{ref}}^{\mathrm{wt}}+\boldsymbol{E}_{\mathbf{I I}}^{\mathbf{d}},
$$

with

$\boldsymbol{A}_{\mathbf{I I}}^{\mathbf{d}}=e^{\boldsymbol{A}_{\mathbf{I I}} t_{\mathrm{s}}}, \boldsymbol{B}_{\mathbf{I I}}^{\mathbf{d}}=\int_{0}^{t_{\mathrm{s}}}\left(e^{\boldsymbol{A}_{\mathbf{I I}} t} \boldsymbol{B}_{\mathbf{I I}}\right) d t, \boldsymbol{E}_{\mathbf{I I}}^{\mathbf{d}}=\int_{0}^{t_{\mathrm{s}}}\left(e^{\boldsymbol{A}_{\mathbf{I I}} t} \boldsymbol{E}_{\mathbf{I I}}\right) d t$.

B. Calculation of $\frac{\partial T_{\mathrm{s}}}{\partial P_{\mathrm{ref}}^{\mathrm{wt}}}$

The shaft torque $T_{\mathrm{s}}$ twists the low-speed shaft. According to the motion equation,

$$
\dot{\omega}_{\mathrm{r}}=\frac{1}{J_{\mathrm{r}}}\left(T_{\mathrm{a}}-T_{\mathrm{s}}\right) \Rightarrow T_{\mathrm{s}}=T_{\mathrm{a}}-J_{\mathrm{r}} \omega_{\mathrm{r}}
$$

Substituting (3) and (4) into (26),

$$
\begin{aligned}
T_{\mathrm{s}}=T_{\mathrm{a}}-J_{\mathrm{r}} \dot{\omega}_{\mathrm{r}} & =T_{\mathrm{a}}-\frac{J_{\mathrm{r}}}{J_{\mathrm{t}}}\left(T_{\mathrm{a}}-\eta_{\mathrm{g}} T_{\mathrm{g}}\right) \\
& =\frac{J_{\mathrm{t}}-J_{\mathrm{r}}}{J_{\mathrm{t}}} T_{\mathrm{a}}+\frac{\eta_{\mathrm{g}} J_{\mathrm{r}}}{J_{\mathrm{t}}} T_{\mathrm{g}} \\
& =\frac{\eta_{\mathrm{g}}^{2} J_{\mathrm{g}}}{J_{\mathrm{t}}} T_{\mathrm{a}}+\frac{\eta_{\mathrm{g}} J_{\mathrm{r}}}{J_{\mathrm{t}}} T_{\mathrm{g}} .
\end{aligned}
$$

Accordingly,

$$
\Delta T_{\mathrm{s}}=\frac{\eta_{\mathrm{g}}^{2} J_{\mathrm{g}}}{J_{\mathrm{t}}} \Delta T_{\mathrm{a}}+\frac{\eta_{\mathrm{g}} J_{\mathrm{r}}}{J_{\mathrm{t}}} \Delta T_{\mathrm{g}} .
$$

During the sampling period, the operation region of the WT may stay or change to another. When the current operating point is in Region I, i.e., $\theta_{0}>0$, with the increase of $\Delta P_{\mathrm{ref}}^{\mathrm{wt}}$, $\theta_{0}+\Delta \theta$ will decrease. If $\theta_{0}+\Delta \theta=0$, the system will transit into Region II. The corresponding $\Delta P_{\text {ref }}^{\mathrm{wt}}$ is defined by $\Delta P_{\mathrm{I} \rightarrow \mathrm{II}}^{\mathrm{wt}}$, which can be considered as the threshold.
According to (24),

$$
\Delta \beta=\boldsymbol{B}_{\mathbf{I}}^{\mathrm{d}}(2,1) \Delta P_{\mathrm{ref}}^{\mathrm{wt}}+\boldsymbol{E}_{\mathbf{I}}^{\mathrm{d}}(2, \mathbf{1}),
$$

where $B_{\mathrm{I}}^{\mathrm{d}}(\mathbf{2}, \mathbf{1})$ and $\boldsymbol{E}_{\mathrm{I}}^{\mathrm{d}}(\mathbf{2}, \mathbf{1})$ are the matrix elements of $\boldsymbol{B}_{\mathrm{I}}^{\mathrm{d}}$ and $\boldsymbol{E}_{\mathbf{I}}^{\mathbf{d}}$, respectively. Since $\frac{\Delta \beta}{\Delta \theta} \approx \frac{\partial \beta}{\partial \theta}=K_{0}+2 K_{\theta} \theta_{0}$,

$$
\Delta \theta=\frac{\boldsymbol{B}_{\mathbf{I}}^{\mathrm{d}}(\mathbf{2}, \mathbf{1}) \Delta P_{\mathrm{ref}}^{\mathrm{wt}}+\boldsymbol{E}_{\mathbf{I}}^{\mathrm{d}}(\mathbf{2}, \mathbf{1})}{K_{0}+2 K_{\theta} \theta_{0}} .
$$

According to $\Delta \theta=-\theta_{0}, \Delta P_{\mathrm{I} \rightarrow \mathrm{II}}^{\mathrm{wt}}$ can be derived by,

$$
\Delta P_{\mathrm{I} \rightarrow \mathrm{II}}^{\mathrm{wt}}=\frac{-\theta_{0}\left(K_{0}+2 K_{\theta} \theta_{0}\right)-\boldsymbol{E}_{\mathrm{I}}^{\mathrm{d}}(\mathbf{2}, \mathbf{1})}{\boldsymbol{B}_{\mathrm{I}}^{\mathrm{d}}(\mathbf{2}, \mathbf{1})} .
$$

When the current operating point is in Region II, i.e., $\theta_{0}=$ 0 , with the decrease of $\Delta P_{\mathrm{ref}}^{\mathrm{wt}}, \omega_{\mathrm{f} 0}+\Delta \omega_{\mathrm{f}}$ will increase. If $\omega_{\mathrm{f} 0}+\Delta \omega_{\mathrm{f}}=\omega_{\mathrm{g} \_ \text {rated }}$, the system will transit into Region I. The corresponding $\Delta P_{\text {ref }}^{\mathrm{wt}}$ is defined by $\Delta P_{\mathrm{II} \rightarrow \mathrm{I}}^{\mathrm{wt}}$, which can be considered as the threshold.

According to (25),

$$
\Delta \omega_{\mathrm{f}}=B_{\mathrm{II}}^{\mathrm{d}}(2,1) \Delta P_{\mathrm{ref}}^{\mathrm{wt}}+\boldsymbol{E}_{\mathrm{II}}^{\mathrm{d}}(2,1),
$$

where $B_{\mathrm{II}}^{\mathrm{d}}(2,1)$ and $E_{\mathrm{II}}^{\mathrm{d}}(2,1)$ are the matrix elements of $\boldsymbol{B}_{\mathrm{II}}^{\mathrm{d}}$ and $\boldsymbol{E}_{\mathrm{II}}^{\mathrm{d}}$, respectively. by,

According to $\omega_{\mathrm{f} 0}+\Delta \omega_{\mathrm{f}}=\omega_{\mathrm{g} \_ \text {rated }}, \Delta P_{\mathrm{II} \rightarrow \mathrm{I}}^{\mathrm{wt}}$ can be derived

$$
\Delta P_{\mathrm{II} \rightarrow \mathrm{I}}^{\mathrm{wt}}=\frac{\omega_{\mathrm{g} \_ \text {rated }}-\omega_{\mathrm{f} 0}-\boldsymbol{E}_{\mathrm{II}}^{\mathrm{d}}(2,1)}{\boldsymbol{B}_{\mathrm{II}}^{\mathrm{d}}(2, \mathbf{1})} .
$$

In the following, 4 cases are summarized to calculate $\frac{\partial T_{\mathrm{s}}}{\partial P_{\mathrm{ref}}^{\mathrm{wt}}}$.

Case 1: Region $\mathbf{I} \rightarrow$ Region $\mathbf{I}$.

If $\Delta P_{\text {ref }}^{\mathrm{wt}} \leq \Delta P_{\mathrm{I} \rightarrow \mathrm{II}}^{\mathrm{wt}}$, the system stays in Region I. Based on (15) and (16), (28) can be transformed into,

$$
\Delta T_{\mathrm{s}}=\boldsymbol{C}_{\mathbf{I}}^{\boldsymbol{T}_{\mathrm{s}}} \Delta x+\boldsymbol{D}_{\mathbf{I}}^{\boldsymbol{T}_{\mathrm{s}}} \Delta P_{\mathrm{ref}}^{\mathrm{wt}},
$$

with

$$
\begin{aligned}
\boldsymbol{C}_{\mathbf{I}}^{\boldsymbol{T}_{\mathrm{s}}} & =\left[\frac{\eta_{\mathrm{g}}^{2} J_{\mathrm{g}}}{J_{\mathrm{t}}} \frac{\partial T_{\mathrm{a}}}{\partial \omega_{\mathrm{g}}} \quad \frac{\eta_{\mathrm{g}}^{2} J_{\mathrm{g}}}{J_{\mathrm{t}}} \frac{\partial T_{\mathrm{a}}}{\partial \beta}-\frac{P_{\mathrm{g} 0} \eta_{\mathrm{g}} J_{\mathrm{r}}}{\omega_{\mathrm{f} 0}^{2} J_{\mathrm{t}}}\right], \\
\boldsymbol{D}_{\mathbf{I}}^{\boldsymbol{T}_{\mathbf{s}}} & =\frac{\eta_{\mathrm{g}} J_{\mathrm{r}}}{J_{\mathrm{t}} \omega_{\mathrm{f} 0}} .
\end{aligned}
$$

Based on (24) and (34),

$$
\Delta T_{\mathrm{s}}(k+1)=\left(\boldsymbol{C}_{\mathbf{I}}^{\boldsymbol{T}_{\mathrm{s}}} \boldsymbol{B}_{\mathbf{I}}^{\mathbf{d}}+\boldsymbol{D}_{\mathbf{I}}^{\boldsymbol{T}_{\mathrm{s}}}\right) \Delta P_{\mathrm{ref}}^{\mathrm{wt}}+\boldsymbol{C}_{\mathbf{I}}^{\boldsymbol{T}_{\mathrm{s}}} \boldsymbol{E}_{\mathbf{I}}^{\mathbf{d}} .
$$

Hence,

$$
\frac{\partial T_{\mathrm{s}}}{\partial P_{\mathrm{ref}}^{\mathrm{wt}}} \approx \frac{\Delta T_{\mathrm{s}}}{\Delta P_{\mathrm{ref}}^{\mathrm{wt}}}=S_{1}^{T_{\mathrm{s}}}+\frac{Y_{1}^{T_{\mathrm{s}}}}{\Delta P_{\mathrm{ref}}^{\mathrm{wt}}},
$$

with $S_{1}^{T_{\mathrm{s}}}=\boldsymbol{C}_{\mathrm{I}}^{\boldsymbol{T}_{\mathrm{s}}} \boldsymbol{B}_{\mathrm{I}}^{\mathrm{d}}+\boldsymbol{D}_{\mathrm{I}}^{\boldsymbol{T}_{\mathrm{s}}}, Y_{1}^{T_{\mathrm{s}}}=\boldsymbol{C}_{\mathrm{I}}^{\boldsymbol{T}_{\mathrm{s}}} \boldsymbol{E}_{\mathrm{I}}^{\mathrm{d}}$. The subscript of $S_{1}^{T_{\mathrm{s}}}$ and $Y_{1}^{T_{\mathrm{s}}}$ indicates the case index.

\section{Case 2: Region II $\rightarrow$ Region II.}

If $\Delta P_{\mathrm{ref}}^{\mathrm{wt}}>\Delta P_{\mathrm{II} \rightarrow \mathrm{I}}^{\mathrm{wt}}$, the system stays in Region II. Based on (15) and (16), (28) can be transformed into,

$$
\Delta T_{\mathrm{s}}=\boldsymbol{C}_{\mathbf{I I}}^{\boldsymbol{T}_{\mathrm{s}}} \Delta x+\boldsymbol{D}_{\mathrm{II}}^{\boldsymbol{T}_{\mathrm{s}}} \Delta P_{\mathrm{ref}}^{\mathrm{wt}},
$$

with

$$
\boldsymbol{C}_{\mathbf{I I}}^{\boldsymbol{T}_{\mathrm{s}}}=\left[\frac{\eta_{\mathrm{g}}^{2} J_{\mathrm{g}}}{J_{\mathrm{t}}} \frac{\partial T_{\mathrm{a}}}{\partial \omega_{\mathrm{g}}}-\frac{P_{\mathrm{g} 0} \eta_{\mathrm{g}} J_{\mathrm{r}}}{\omega_{\mathrm{f} 0}^{2} J_{\mathrm{t}}}\right], \boldsymbol{D}_{\mathbf{I I}}^{\boldsymbol{T}_{\mathrm{s}}}=\frac{\eta_{\mathrm{g}} J_{\mathrm{r}}}{J_{\mathrm{t}} \omega_{\mathrm{f} 0}} .
$$


Based on (25) and (37),

$$
\Delta T_{\mathrm{s}}(k+1)=\left(\boldsymbol{C}_{\mathbf{I I}}^{\boldsymbol{T}_{\mathrm{s}}} \boldsymbol{B}_{\mathrm{II}}^{\mathrm{d}}+\boldsymbol{D}_{\mathrm{II}}^{\boldsymbol{T}_{\mathrm{s}}}\right) \Delta P_{\mathrm{ref}}^{\mathrm{wt}}+\boldsymbol{C}_{\mathbf{I I}}^{\boldsymbol{T}_{\mathrm{s}}} \boldsymbol{E}_{\mathbf{I I}}^{\mathrm{d}} .
$$

Hence,

$$
\frac{\partial T_{\mathrm{s}}}{\partial P_{\mathrm{ref}}^{\mathrm{wt}}} \approx \frac{\Delta T_{\mathrm{s}}}{\Delta P_{\mathrm{ref}}^{\mathrm{wt}}}=S_{2}^{T_{\mathrm{s}}}+\frac{Y_{2}^{T_{\mathrm{s}}}}{\Delta P_{\mathrm{ref}}^{\mathrm{wt}}},
$$

with $S_{2}^{T_{\mathrm{s}}}=\boldsymbol{C}_{\mathrm{II}}^{\boldsymbol{T}_{\mathrm{s}}} \boldsymbol{B}_{\mathrm{II}}^{\mathrm{d}}+\boldsymbol{D}_{\mathrm{II}}^{\boldsymbol{T}_{\mathrm{s}}}, Y_{2}^{T_{\mathrm{s}}}=\boldsymbol{C}_{\mathrm{II}}^{\boldsymbol{T}_{\mathrm{s}}} \boldsymbol{E}_{\mathrm{II}}^{\mathrm{d}}$.

\section{Case 3: Region I $\rightarrow$ Region II.}

If $\Delta P_{\mathrm{ref}}^{\mathrm{wt}}>\Delta P_{\mathrm{I} \rightarrow \mathrm{II}}^{\mathrm{wt}}$, the system transits from Region I to Region II. In that case, $\Delta P_{\text {ref }}^{\text {wt }}$ could be divided into two parts: $\Delta P_{\mathrm{I} \rightarrow \mathrm{II}}^{\mathrm{wt}}$ and $\Delta P_{\mathrm{ref}}^{\mathrm{wt}}-\Delta P_{\mathrm{I} \rightarrow \mathrm{II}}^{\mathrm{wt}}$. The former part works in Region I and the latter part works in Region II.

Accordingly, $\Delta T_{\mathrm{s}}(k+1)$ can be approximately calculated by,

$$
\begin{aligned}
\Delta T_{\mathrm{s}}(k+1) \approx & S_{1}^{T_{\mathrm{s}}} \Delta P_{\mathrm{I} \rightarrow \mathrm{II}}^{\mathrm{wt}}+Y_{1}^{T_{\mathrm{s}}} \\
& +S_{2}^{T_{\mathrm{s}}}\left(\Delta P_{\mathrm{ref}}^{\mathrm{wt}}-\Delta P_{\mathrm{I} \rightarrow \mathrm{II}}^{\mathrm{wt}}\right)+Y_{2}^{T_{\mathrm{s}}} \\
\approx & S_{2}^{T_{\mathrm{s}}} \Delta P_{\mathrm{ref}}^{\mathrm{wt}}+Y_{1}^{T_{\mathrm{s}}}+Y_{2}^{T_{\mathrm{s}}}+\left(S_{1}^{T_{\mathrm{s}}}-S_{2}^{T_{\mathrm{s}}}\right) \Delta P_{\mathrm{I} \rightarrow \mathrm{II}}^{\mathrm{wt}} .
\end{aligned}
$$

Hence,

$$
\frac{\partial T_{\mathrm{s}}}{\partial P_{\mathrm{ref}}^{\mathrm{wt}}} \approx \frac{\Delta T_{\mathrm{s}}}{\Delta P_{\mathrm{ref}}^{\mathrm{wt}}}=S_{3}^{T_{\mathrm{s}}}+\frac{Y_{3}^{T_{\mathrm{s}}}}{\Delta P_{\mathrm{ref}}^{\mathrm{wt}}},
$$

with $S_{3}^{T_{\mathrm{s}}}=S_{2}^{T_{\mathrm{s}}}, Y_{3}^{T_{\mathrm{s}}}=Y_{1}^{T_{\mathrm{s}}}+Y_{2}^{T_{\mathrm{s}}}+\left(S_{1}^{T_{\mathrm{s}}}-S_{2}^{T_{\mathrm{s}}}\right) \Delta P_{\mathrm{I} \rightarrow \mathrm{II}}^{\mathrm{wt}}$.

\section{Case 4: Region II $\rightarrow$ Region I.}

If $\Delta P_{\text {ref }}^{\mathrm{wt}} \leq \Delta P_{\mathrm{II} \rightarrow \mathrm{I}}^{\mathrm{wt}}$, the system transits from Region II to Region I. Similarly, $\Delta P_{\text {ref }}^{\text {wt }}$ could be divided into two parts: $\Delta P_{\mathrm{II} \rightarrow \mathrm{I}}^{\mathrm{wt}}$ and $\Delta P_{\mathrm{ref}}^{\mathrm{wt}}-\Delta P_{\mathrm{II} \rightarrow \mathrm{I}}^{\mathrm{wt}}$. The former part works in Region II and the latter part works in Region I. $\Delta T_{\mathrm{S}}(k+1)$ can be approximately calculated by,

$$
\begin{aligned}
\Delta T_{\mathrm{s}}(k+1) \approx & S_{2}^{T_{\mathrm{s}}} \Delta P_{\mathrm{II} \rightarrow \mathrm{I}}^{\mathrm{wt}}+Y_{2}^{T_{\mathrm{s}}} \\
& +S_{1}^{T_{\mathrm{s}}}\left(\Delta P_{\mathrm{ref}}^{\mathrm{wt}}-\Delta P_{\mathrm{II} \rightarrow \mathrm{I}}^{\mathrm{wt}}\right)+S_{1}^{T_{\mathrm{s}}} \\
\approx & S_{1}^{T_{\mathrm{s}}} \Delta P_{\mathrm{ref}}^{\mathrm{wt}}+Y_{2}^{T_{\mathrm{s}}}+Y_{1}^{T_{\mathrm{s}}}+\left(S_{2}^{T_{\mathrm{s}}}-S_{1}^{T_{\mathrm{s}}}\right) \Delta P_{\mathrm{II} \rightarrow \mathrm{I}}^{\mathrm{wt}}
\end{aligned}
$$

Hence,

$$
\frac{\partial T_{\mathrm{s}}}{\partial P_{\mathrm{ref}}^{\mathrm{wt}}} \approx \frac{\Delta T_{\mathrm{s}}}{\Delta P_{\mathrm{ref}}^{\mathrm{wt}}}=S_{4}^{T_{\mathrm{s}}}+\frac{Y_{4}^{T_{\mathrm{s}}}}{\Delta P_{\mathrm{ref}}^{\mathrm{wt}}},
$$

with $S_{4}^{T_{\mathrm{s}}}=S_{1}^{T_{\mathrm{s}}}, Y_{4}^{T_{\mathrm{s}}}=Y_{2}^{T_{\mathrm{s}}}+Y_{1}^{T_{\mathrm{s}}}+\left(S_{2}^{T_{\mathrm{s}}}-S_{1}^{T_{\mathrm{s}}}\right) \Delta P_{\mathrm{II} \rightarrow \mathrm{I}}^{\mathrm{wt}}$.

In the following, for generality, the subscripts of $S^{T_{\mathrm{s}}}$ and $Y^{T_{\mathrm{s}}}$ are omitted. Based on the measurements, $S^{T_{\mathrm{s}}}$ and $Y^{T_{\mathrm{s}}}$ can be calculated explicitly, which are sent to the wind farm controller to formulate the optimal dispatch algorithm.

\section{Calculation of $\frac{\partial F_{\mathrm{t}}}{\partial P_{\mathrm{ref}}^{\mathrm{wt}}}$}

Similar to $\frac{\partial T_{\mathrm{s}}}{\partial P_{\mathrm{ref}}^{\mathrm{wt}}}, 4$ cases are summarized to calculate $\frac{\partial F_{\mathrm{t}}}{\partial P_{\mathrm{ref}}^{\mathrm{wt}}}$.

Case 1: Region $\mathbf{I} \rightarrow$ Region $\mathbf{I}$.

If $\Delta P_{\text {ref }}^{\mathrm{wt}} \leq \Delta P_{\mathrm{I} \rightarrow \mathrm{II}}^{\mathrm{wt}}$, the system stays in Region I. $F_{\mathrm{t}}$ can be calculated by,

$$
\Delta F_{\mathrm{t}}=\boldsymbol{C}_{\mathbf{I}}^{\boldsymbol{F}_{\mathrm{t}}} x
$$

with $\boldsymbol{C}_{\mathbf{I}}^{\boldsymbol{F}_{\mathbf{t}}}=\left[\begin{array}{lll}\frac{\partial F_{\mathrm{t}}}{\partial \omega_{\mathrm{g}}} & \frac{\partial F_{\mathrm{t}}}{\partial \beta} & 0\end{array}\right]$.
Based on (24) and (44),

$$
\Delta F_{\mathrm{t}}(k+1)=\boldsymbol{C}_{\mathbf{I}}^{\boldsymbol{F}_{\mathrm{t}}} \boldsymbol{B}_{\mathbf{I}}^{\mathrm{d}} \Delta P_{\mathrm{ref}}^{\mathrm{wt}}+\boldsymbol{C}_{\mathbf{I}}^{\boldsymbol{F}_{\mathrm{t}}} \boldsymbol{E}_{\mathbf{I}}^{\mathrm{d}} .
$$

Hence,

$$
\frac{\partial F_{\mathrm{t}}}{\partial P_{\mathrm{ref}}^{\mathrm{wt}}} \approx \frac{\Delta F_{\mathrm{t}}}{\Delta P_{\mathrm{ref}}^{\mathrm{wt}}}=S_{1}^{F_{\mathrm{t}}}+\frac{Y_{1}^{F_{\mathrm{t}}}}{\Delta P_{\mathrm{ref}}^{\mathrm{wt}}},
$$

with $S_{1}^{F_{\mathrm{t}}}=\boldsymbol{C}_{\mathbf{I}}^{\boldsymbol{F}_{\mathrm{t}}} \boldsymbol{B}_{\mathbf{I}}^{\mathrm{d}}, Y_{1}^{F_{\mathrm{t}}}=\boldsymbol{C}_{\mathbf{I}}^{\boldsymbol{F}_{\mathrm{t}}} \boldsymbol{E}_{\mathbf{I}}^{\mathrm{d}}$. The subscript of $S_{1}^{F_{\mathrm{s}}}$ and $Y_{1}^{F_{\mathrm{s}}}$ indicates the case index.

\section{Case 2: Region II $\rightarrow$ Region II.}

If $\Delta P_{\mathrm{ref}}^{\mathrm{wt}}>\Delta P_{\mathrm{II} \rightarrow \mathrm{I}}^{\mathrm{wt}}$, the system stays in Region II. $F_{\mathrm{t}}$ can be calculated by,

$$
\Delta F_{\mathrm{t}}=C_{\mathbf{I I}}^{\boldsymbol{F}_{\mathbf{t}}} x
$$

with $\boldsymbol{C}_{\mathbf{I I}}^{\boldsymbol{F}_{\mathbf{t}}}=\left[\begin{array}{ll}\frac{\partial F_{\mathrm{t}}}{\partial \omega_{g}} & 0\end{array}\right]$.

Based on (25) and (47),

$$
\Delta F_{\mathrm{t}}(k+1)=\boldsymbol{C}_{\mathbf{I I}}^{\boldsymbol{F}_{\mathrm{t}}} \boldsymbol{B}_{\mathrm{II}}^{\mathrm{d}} \Delta P_{\mathrm{ref}}^{\mathrm{wt}}+\boldsymbol{C}_{\mathbf{I I}}^{\boldsymbol{F}_{\mathrm{t}}} \boldsymbol{E}_{\mathbf{I I}}^{\mathrm{d}} .
$$

Hence,

$$
\frac{\partial F_{\mathrm{t}}}{\partial P_{\mathrm{ref}}^{\mathrm{wt}}} \approx \frac{\Delta F_{\mathrm{t}}}{\Delta P_{\mathrm{ref}}^{\mathrm{wt}}}=S_{2}^{F_{\mathrm{t}}}+\frac{Y_{2}^{F_{\mathrm{t}}}}{\Delta P_{\mathrm{ref}}^{\mathrm{wt}}},
$$

with $S_{2}^{F_{\mathrm{t}}}=\boldsymbol{C}_{\mathbf{I I}}^{\boldsymbol{F}_{\mathrm{t}}} \boldsymbol{B}_{\mathbf{I I}}^{\mathrm{d}}, Y_{1}^{F_{\mathrm{t}}}=\boldsymbol{C}_{\mathbf{I I}}^{\boldsymbol{F}_{\mathrm{t}}} \boldsymbol{E}_{\mathbf{I I}}^{\mathrm{d}}$.

\section{Case 3: Region I $\rightarrow$ Region II.}

If $\Delta P_{\mathrm{ref}}^{\mathrm{wt}}>\Delta P_{\mathrm{I} \rightarrow \mathrm{II}}^{\mathrm{wt}}$, the system transits from Region I to Region II. $\Delta F_{\mathrm{t}}(k+1)$ can be approximately calculated by,

$$
\begin{aligned}
\Delta F_{\mathrm{t}}(k+1) \approx & S_{1}^{F_{\mathrm{t}}} \Delta P_{\mathrm{I} \rightarrow \mathrm{II}}^{\mathrm{wt}}+Y_{1}^{F_{\mathrm{t}}} \\
& +S_{2}^{F_{\mathrm{t}}}\left(\Delta P_{\mathrm{ref}}^{\mathrm{wt}}-\Delta P_{\mathrm{I} \rightarrow \mathrm{II}}^{\mathrm{wt}}\right)+Y_{2}^{F_{\mathrm{t}}} \\
\approx & S_{2}^{F_{\mathrm{t}}} \Delta P_{\mathrm{ref}}^{\mathrm{wt}}+Y_{1}^{F_{\mathrm{t}}}+Y_{2}^{F_{\mathrm{t}}}+\left(S_{1}^{F_{\mathrm{t}}}-S_{2}^{F_{\mathrm{t}}}\right) \Delta P_{\mathrm{I} \rightarrow \mathrm{II}}^{\mathrm{wt}} \cdot
\end{aligned}
$$

Hence,

$$
\frac{\partial F_{\mathrm{t}}}{\partial P_{\mathrm{ref}}^{\mathrm{wt}}} \approx \frac{\Delta F_{\mathrm{t}}}{\Delta P_{\mathrm{ref}}^{\mathrm{wt}}}=S_{3}^{F_{\mathrm{t}}}+\frac{Y_{3}^{F_{\mathrm{t}}}}{\Delta P_{\mathrm{ref}}^{\mathrm{wt}}},
$$

with $S_{3}^{F_{\mathrm{t}}}=S_{2}^{F_{\mathrm{t}}}, Y_{3}^{F_{\mathrm{t}}}=Y_{1}^{F_{\mathrm{t}}}+Y_{2}^{F_{\mathrm{t}}}+\left(S_{1}^{F_{\mathrm{t}}}-S_{2}^{F_{\mathrm{t}}}\right) \Delta P_{\mathrm{I} \rightarrow \mathrm{II}}^{\mathrm{wt}}$.

\section{Case 4: Region II $\rightarrow$ Region I.}

If $\Delta P_{\mathrm{ref}}^{\mathrm{wt}} \leq \Delta P_{\mathrm{II} \rightarrow \mathrm{I}}^{\mathrm{wt}}$, the system transits from Region II to Region I. $\Delta F_{\mathrm{t}}(k+1)$ can be approximately calculated by,

$$
\begin{aligned}
\Delta F_{\mathrm{t}}(k+1) \approx & S_{2}^{F_{\mathrm{t}}} \Delta P_{\mathrm{II} \rightarrow \mathrm{I}}^{\mathrm{wt}}+Y_{2}^{F_{\mathrm{t}}} \\
& +S_{1}^{F_{\mathrm{t}}}\left(\Delta P_{\mathrm{ref}}^{\mathrm{wt}}-\Delta P_{\mathrm{II} \rightarrow \mathrm{I}}^{\mathrm{wt}}\right)+S_{1}^{F_{\mathrm{t}}} \\
\approx & S_{1}^{F_{\mathrm{t}}} \Delta P_{\mathrm{ref}}^{\mathrm{wt}}+Y_{2}^{F_{\mathrm{t}}}+Y_{1}^{F_{\mathrm{t}}}+\left(S_{2}^{F_{\mathrm{t}}}-S_{1}^{F_{\mathrm{t}}}\right) \Delta P_{\mathrm{II} \rightarrow \mathrm{I}}^{\mathrm{wt}}
\end{aligned}
$$

Hence,

$$
\frac{\partial F_{\mathrm{t}}}{\partial P_{\mathrm{ref}}^{\mathrm{wt}}} \approx \frac{\Delta F_{\mathrm{t}}}{\Delta P_{\mathrm{ref}}^{\mathrm{wt}}}=S_{4}^{F_{\mathrm{t}}}+\frac{Y_{4}^{F_{\mathrm{t}}}}{\Delta P_{\mathrm{ref}}^{\mathrm{wt}}},
$$

with $S_{4}^{F_{\mathrm{t}}}=S_{1}^{F_{\mathrm{t}}}, Y_{4}^{F_{\mathrm{t}}}=Y_{2}^{F_{\mathrm{t}}}+Y_{1}^{F_{\mathrm{t}}}+\left(S_{2}^{F_{\mathrm{t}}}-S_{1}^{F_{\mathrm{t}}}\right) \Delta P_{\mathrm{II} \rightarrow \mathrm{I}}^{\mathrm{wt}}$.

According to (2), $\frac{\partial F_{\mathrm{t}}}{\partial \omega_{\mathrm{g}}}$ and $\frac{\partial F_{\mathrm{t}}}{\partial \beta}$ in (44) and (47) are the functions of $\frac{\partial C_{\mathrm{t}}}{\partial \omega_{\mathrm{g}}}$ and $\frac{\partial C_{\mathrm{t}}}{\partial \beta}$, respectively, i.e.,

$$
\frac{\partial F_{\mathrm{t}}}{\partial \omega_{\mathrm{g}}}=\frac{P_{0}}{v_{\mathrm{r} 0}} \frac{\partial C_{\mathrm{t}}}{\partial \omega_{\mathrm{g}}}, \frac{\partial F_{\mathrm{t}}}{\partial \beta}=\frac{P_{0}}{v_{\mathrm{r} 0}} \frac{\partial C_{\mathrm{t}}}{\partial \beta} .
$$


The function $C_{\mathrm{t}}$ is nonlinear. Similar to $C_{\mathrm{p}}, C_{\mathrm{t}}$ is also described in a lookup table derived from the geometry of the blades with inputs $\lambda$ and $\theta$ [21]. The corresponding data for this study can be obtained in the wind turbine model of SimWindFarm. The plot of $C_{\mathrm{t}}(\lambda, \theta)$ based on the lookup table is shown in Fig. 6.

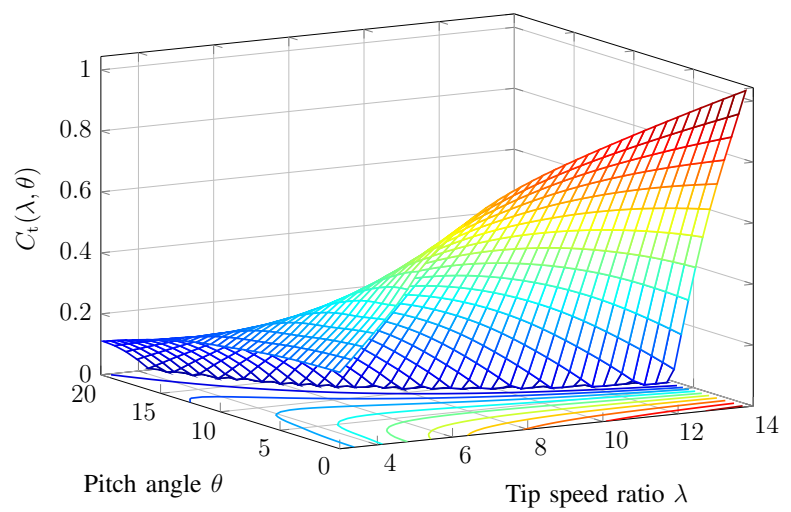

Fig. 6. $C_{\mathrm{t}}(\lambda, \theta)$ based on the data from SimWindFarm [21].

According to $v_{\mathrm{r} 0}, \omega_{\mathrm{g} 0}$, and $\theta_{0}$, the power coefficient at the operating point $C_{\mathrm{t} 0}$ can be found based on the lookup table, i.e., $C_{\mathrm{t} 0}=C_{\mathrm{t}(n, m)}$, where $n$ and $m$ are the corresponding row and column indices, respectively. $\frac{\partial C_{t}}{\partial \omega_{\mathrm{g}}}$ and $\frac{\partial C_{\mathrm{t}}}{\partial \beta}$ can be calculated by,

$$
\begin{gathered}
\frac{\partial C_{\mathrm{t}}}{\partial \omega_{\mathrm{g}}}=\frac{R \eta_{\mathrm{g}}}{v_{\mathrm{r} 0}} \frac{\partial C_{\mathrm{t}}}{\partial \lambda}, \frac{\partial C_{\mathrm{t}}}{\partial \lambda} \approx \frac{C_{\mathrm{t}(n, m+1)}-C_{\mathrm{t} 0}}{\Delta \lambda_{\mathrm{tab}}}, \\
\frac{\partial C_{\mathrm{t}}}{\partial \beta}=\frac{\partial C_{\mathrm{t}}}{\partial \theta} \frac{1}{K_{0}+2 K_{\theta} \theta_{0}}, \frac{\partial C_{\mathrm{t}}}{\partial \theta} \approx \frac{C_{\mathrm{t}(n+1, m)}-C_{\mathrm{t} 0}}{\Delta \theta_{\mathrm{tab}}} .
\end{gathered}
$$

In the following, for generality, the subscripts of $S^{F_{\mathrm{t}}}$ and $Y^{F_{\mathrm{t}}}$ are omitted. Based on the measurements, $S^{F_{\mathrm{t}}}$ and $Y^{F_{\mathrm{t}}}$ can be calculated explicitly, which are sent to the wind farm controller to formulate the optimal dispatch algorithm.

\section{Formulation of Optimal Dispatch AlgORithm}

\section{A. Cost Function}

Conventionally, the dispatch algorithm is based on a proportional distribution of the availabe active power [7]. Suppose a wind farm consists of $N_{\mathrm{wt}}$ WTs. According to [7], the power reference of the $i$ th WT is calculated by,

$$
P_{\mathrm{ref}}^{\mathrm{wt} i}=\alpha_{i} P_{\mathrm{ref}}^{\mathrm{wfc}}, \quad \alpha_{i}=\frac{P_{\mathrm{avi}}^{\mathrm{wt} i} i}{P_{\mathrm{avi}}^{\mathrm{wfc}}}, \quad P_{\mathrm{avi}}^{\mathrm{wfc}}=\sum_{i=1}^{N_{\mathrm{wt}}} P_{\mathrm{avi}}^{\mathrm{wt} i},
$$

where $\alpha_{i}$ is the distribution factor of the $i$ th WT and $P_{\mathrm{avi}}^{\mathrm{wt}}{ }^{i}$ is the available power of the $i$ th WT.

In this study, based on the proportional dispatch algorithm, the control objective is to dynamically redistribute power to minimize the fatigue loads experienced by the WTs while tracking $P_{\text {ref }}^{\mathrm{wfc}}$ all the time. The $P_{\text {ref }}^{\mathrm{wfc}}$ tracking is regarded as an equality constraint, described in Section VI-B. The minimization of the fatigue loads implies the minimization of the variation of $T_{\mathrm{s}}$ and $F_{\mathrm{t}}$, which is included in the cost function.
By defining the control vector $u_{\text {ref }}$,

$$
u_{\mathrm{ref}} \triangleq\left[\Delta P_{\mathrm{ref}}^{\mathrm{wt} \_}, \cdots, \Delta P_{\mathrm{ref}}^{\mathrm{wt}-N_{\mathrm{wt}}}\right]^{\prime}, u_{\mathrm{ref}} \in \mathbb{R}^{N_{\mathrm{wt}} \times 1},
$$

the optimization problem can be formulated as,

$$
\begin{aligned}
& \min _{u_{\mathrm{ref}}} \sum_{i=1}^{N_{\mathrm{wt}}} \underbrace{\left\|u_{\mathrm{ref}}^{i}+P_{\mathrm{g} 0}^{i}-\alpha_{i} P_{\mathrm{ref}}^{\mathrm{wfc}}\right\|_{Q_{P}}^{2}}_{\text {Term 1 }} \\
& +\underbrace{\left\|\frac{\partial T_{\mathrm{s}}^{i}}{\partial P_{\text {ref }}^{\mathrm{wt} i}} u_{\mathrm{ref}}^{i}\right\|_{Q_{T_{\mathrm{s}}}}^{2}}_{\text {Term 2 }}+\underbrace{\left\|\frac{\partial F_{\mathrm{t}}^{i}}{\partial P_{\mathrm{ref}}^{\mathrm{wt} i}} u_{\mathrm{ref}}^{i}\right\|_{Q_{F_{\mathrm{t}}}}^{2}}_{\text {Term 3 }},
\end{aligned}
$$

where $Q_{P}$ is the weighting factor which penalizes the deviation of $P_{\mathrm{ref}}^{\mathrm{wt} i} i$ to its proportional value, $Q_{T_{\mathrm{s}}}$ is the weighting factor which penalizes the variation of $T_{\mathrm{s}}$, and $Q_{F_{\mathrm{t}}}$ is the weighting factor which penalizes the variation of $F_{\mathrm{t}}$.

As shown in Term 1 of (58), $u_{\mathrm{ref}}^{i}$ is around $\alpha_{i} P_{\mathrm{ref}}^{\mathrm{wfc}}-P_{\mathrm{g} 0}^{i}$. Therefore, by assuming $u_{\mathrm{ref}}^{i} \approx \alpha_{i} P_{\mathrm{ref}}^{\mathrm{wfc}}-P_{\mathrm{g} 0}^{i}$, the case index of the system during the control interval (Case 1 Case 4) in Subsection V-B and Subsection V-C could be estimated. Accordingly, $S^{T_{\mathrm{s}_{-}} i}$ and $S^{Y_{\mathrm{s}_{-}} i}$ could be decided. Term 2 and Term 3 in (58) can be transformed into,

$$
\min _{u_{\mathrm{ref}}} \sum_{i=1}^{N_{\mathrm{wt}}}\left\|S^{T_{\mathrm{s}_{-}} i} u_{\mathrm{ref}}^{i}+Y^{T_{\mathrm{s}_{-}} i}\right\|_{Q_{T_{\mathrm{s}}}}^{2}+\left\|S^{F_{\mathrm{t}_{-}} i} u_{\mathrm{ref}}^{i}+Y^{F_{\mathrm{t}} i}\right\|_{Q_{F_{\mathrm{t}}}}^{2} .
$$

\section{B. Constraints}

The constraints are summarized as follows:

1) Power Reference Tracking: According to $P_{\mathrm{ref}}^{\mathrm{wfc}}$, the total power reference increments of WTs should follow,

$$
\sum_{i=1}^{N_{\mathrm{wt}}} u_{\mathrm{ref}}^{i}=P_{\mathrm{ref}}^{\mathrm{wfc}}-P^{\mathrm{wfc}}
$$

where $P^{\text {wfc }}$ is the measured output of the wind farm.

2) Local WT Constraint: For individual WTs, there exists the available power limit,

$$
u_{\mathrm{ref}} \in\left[-P_{\mathrm{g} 0}, P_{\mathrm{avi}}^{\mathrm{wt}}-P_{\mathrm{g} 0}\right] .
$$

For generality, the turbine index $i$ is omitted.

Besides, as mentioned in Subsection VI-A, the case index of the system is estimated based on $\alpha P_{\text {ref }}^{\mathrm{wfc}}-P_{\mathrm{g} 0}$. Accordingly, $u_{\text {ref }}$ should be limited in the local constraint to guarantee that the system will be in the estimated case, i.e.,

$$
\begin{aligned}
& \left\{\begin{array}{l}
\text { If } \alpha P_{\text {ref }}^{\mathrm{wfc}}-P_{\mathrm{g} 0} \leq \Delta P_{\mathrm{I} \rightarrow \mathrm{II}}^{\mathrm{wt}}\left(\text { Case 1), } u_{\mathrm{ref}} \leq \Delta P_{\mathrm{I} \rightarrow \mathrm{II}}^{\mathrm{wt}},\right. \\
\text { If } \alpha P_{\mathrm{rfc}}^{\mathrm{wfc}}-P_{\mathrm{g} 0}>\Delta P_{\mathrm{It}}^{\mathrm{wt}}\left(\text { Case 2), } u_{\mathrm{ref}}>\Delta P_{\mathrm{II}}^{\mathrm{wt}}\right. \text {, }
\end{array}\right. \\
& \text { If } \alpha P_{\mathrm{ref}}^{\mathrm{wfc}}-P_{\mathrm{g} 0}>\Delta P_{\mathrm{I} \rightarrow \mathrm{II}}^{\mathrm{wt}}\left(\text { Case 3), } u_{\mathrm{ref}}>\Delta P_{\mathrm{I} \rightarrow \mathrm{II}}^{\mathrm{wt}}\right. \text {, } \\
& \text { If } \alpha P_{\mathrm{ref}}^{\mathrm{wfc}}-P_{\mathrm{g} 0} \leq \Delta P_{\mathrm{II} \rightarrow \mathrm{I}}^{\mathrm{wt}}\left(\text { Case 4), } u_{\mathrm{ref}} \leq \Delta P_{\mathrm{II} \rightarrow \mathrm{I}}^{\mathrm{wt}}\right. \text {. }
\end{aligned}
$$

By merging (61) and (62), the feasible operation range of $u_{\text {ref }}$ can be derived,

$$
u_{\mathrm{ref}} \in\left[u_{\min }, u_{\max }\right] .
$$

Accordingly, $P_{\mathrm{min}}^{\mathrm{wt}}=P_{\mathrm{g} 0}+u_{\min }$ and $P_{\max }^{\mathrm{wt}}=P_{\mathrm{g} 0}+u_{\max }$.

The derived optimization problem can be reformulated as a standard Quadratic Programming (QP) problem, whose rank is equal to the number of WTs. It can be efficiently solved by a number of commercial solvers. 


\section{Discussion}

The computation task of the optimal wind farm control mainly consists of formulating and solving the optimal problem. Accordingly, the improvement with the proposed method can be described in the following two aspects.

1) Formulation of the optimal problem: With the proposed method, the operation region can be determined according to the measurements. Accordingly, the offline calculated parameters of the region can be derived and the load sensitivities can be calculated based on the explicit equations. As such, a part of the computation burden required for the online computation can be removed. Moreover, the task has been distributed to local controllers, which can be solved in parallel. With increasing the number of WTs, this efficiency improvement becomes higher. Therefore, the dedicated controller is computationally viable for implementation in large wind farms.

2) Solution of the optimal problem: The time complexity, expressed by $\mathcal{O}$, is used to indicate the amount of time taken by an algorithm. As mentioned in [29], QP problems can be solved roughly with the same efficiency as LP problems. The time complexity of the practical algorithm to solve LP problem with $n$ variables and $m$ constraints requires roughly $\mathcal{O}\left(n^{3} m^{0.5}+n^{2} m^{1.5}\right)$. Compared with the method in [14], the formulated optimal problem of the proposed method has reduced optimization variables $\left(n=N_{\mathrm{wt}}\right)$ and constraints $\left(m=N_{\mathrm{wt}}+1\right)$. The corresponding time complexity of the proposed method is less.

\section{CASE STUdies}

A wind farm comprised of $10 \times 5 \mathrm{MW}$ WTs is used as the test system. The wind field modeling considering turbulences and wake effects for the wind farm is generated from SimWindFarm [21]. The control period of the wind farm control $t_{\mathrm{s}}$ is set as $1 \mathrm{~s}$.

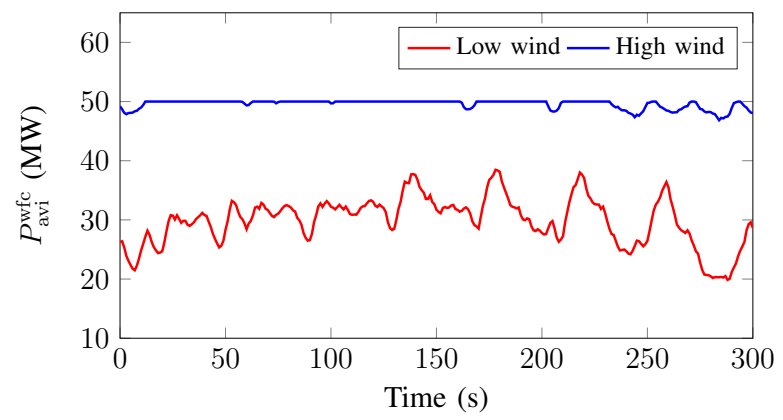

Fig. 7. $P_{\mathrm{avi}}^{\mathrm{wfc}}$ and $P_{\mathrm{wfc}}^{\mathrm{ref}}$ under both low and high wind conditions.

In order to test the efficacy of the developed dispatch algorithm, the operation of the wind farm under both low and high conditions were studied. The available power of the wind farm $P_{\mathrm{avi}}^{\mathrm{wfc}}$ under both conditions is shown in Fig. 7. The typical active control scheme, balance control mode, is used in this study, where the wind farm production is reduced to specified constant levels [30]. The simulation results of the proposed optimal algorithm ("OPT") are compared with the conventional proportional dispatch algorithm in [7] ("CON").
The dynamic turbine behavior in a simulated wind field yields time series of loads or stresses. Through postprocessing, the fatigue cycles based on the rainflow counting method is derived to evaluate the performance of the proposed scheme [31]. Besides, the Damage Equivalent Load (DEL), based on Miner's rule and dependent on materials properties specified by the slope of the S-N curve, is used to quantify the load minimization. In this study, the related calculations are completed by MCrunch, developed by NREL [32].

\section{A. Decision of Weighting Factors}

The control objective of the cost function (58) consists of the following two parts, which represent the minimization of $\Delta T_{\mathrm{s}}$ and $\Delta F_{\mathrm{t}}$, respectively,

$$
\mathrm{Obj} 1 \triangleq \sum_{i=1}^{N_{\mathrm{wt}}}\left\|\Delta T_{\mathrm{s}}^{i}\right\|^{2}, \mathrm{Obj} 2 \triangleq \sum_{i=1}^{N_{\mathrm{wt}}}\left\|\Delta F_{\mathrm{t}}^{i}\right\|^{2} .
$$

The weighting factors for Obj1 and Obj2 are $Q_{T_{\mathrm{s}}}$ and $Q_{F_{\mathrm{t}}}$. They can be considered as a whole part, which represents the load minimization of the wind farm. By defining $Q_{\text {Load }} \triangleq$ $Q_{T_{\mathrm{s}}}+Q_{F_{\mathrm{t}}}$, it is obvious that larger $\frac{Q_{\mathrm{Load}}}{Q_{P}}$ can lead to a better performance of the load alleviation. In this study, $Q_{P}=1$ and $Q_{\text {Load }}=600$. By defining $\gamma \triangleq \frac{Q_{T_{\mathrm{S}}}}{Q_{\text {Load }}}$,

$$
Q_{T_{\mathrm{s}}}=\gamma Q_{\mathrm{Load}}, Q_{F_{\mathrm{t}}}=(1-\gamma) Q_{\mathrm{Load}}
$$

Fig. 8 shows the Pareto frontier under both low and high wind conditions. By increasing the ratio $\gamma$, Obj1 decreases while Obj2 increases. For the low wind condition, it can be observed that $\gamma=0.0017$ is the optimum solution. A significant reduction of $\Delta F_{\mathrm{t}}(\mathrm{Obj} 2)$ can be observed, which is from 1 to $0.33(67 \%)$. Comparably, the reduction of $\Delta T_{\mathrm{s}}$ (Obj1) is not sensitive, which is from 1 to 0.81 (19\%). For the high wind condition, it can be observed that $\gamma=0.003$ is the optimum solution. The reduction of $\Delta F_{\mathrm{t}}(\mathrm{Obj} 2)$ is from 1 to $0.83(17 \%)$. Comparably, the reduction of $\Delta T_{\mathrm{s}}(\mathrm{Obj} 1)$ is similar, which is from 1 to $0.84(16 \%)$.

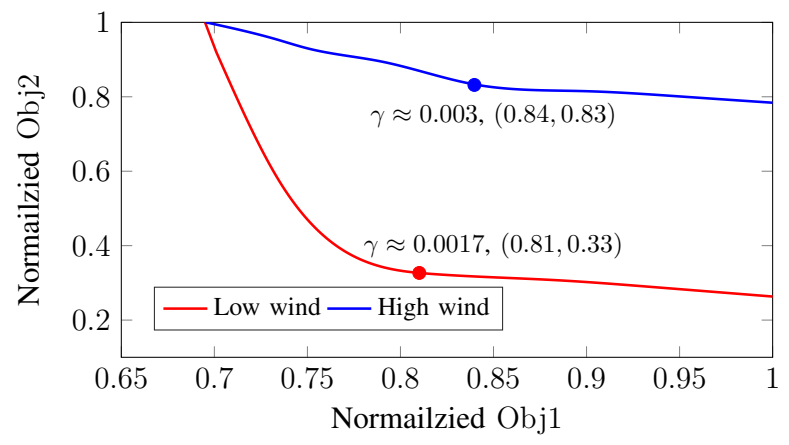

Fig. 8. Pareto frontier under low and high wind conditions.

It can be observed that the optimum $\gamma$ is related to the available power. In this study, $\gamma$ is adaptive to different wind conditions and can be approximately calculated by,

$$
\gamma \approx \frac{\sum_{i=1}^{N_{\mathrm{wt}}} P_{\mathrm{avi}{ }_{\text {max }}{ }_{\mathrm{wt}}^{i}}}{18000}, P_{\mathrm{avi} \_\max }^{\mathrm{wt}}=0.5 \pi \rho R^{2} v_{\mathrm{r}}^{3} C_{\mathrm{p}}^{\max },
$$


where $C_{\mathrm{p}}^{\max }$ is the maximum power coefficient. The unit of $P_{\text {avi max }}^{\mathrm{wt}}$ is in MW. Accordingly, $Q_{T_{\mathrm{s}}}$ and $Q_{F_{\mathrm{t}}}$ can be derived, which are used for the following case scenarios.

\section{B. Operation under Low Wind Condition}

The average wind speeds of all WTs $\left(v_{\text {avr }}\right)$ in the wind farm are listed in Table II, which range from $8.85 \mathrm{~m} / \mathrm{s}$ to $9.97 \mathrm{~m} / \mathrm{s}$. As shown in Fig. 9, three scenarios are defined according to different $P_{\mathrm{ref}}^{\mathrm{wfc}}: P_{\mathrm{ref}}^{\mathrm{wfc}}=20 \mathrm{MW}$ (Scenario 1), $P_{\text {ref }}^{\mathrm{wfc}}=17.5 \mathrm{MW}$ (Scenario 2), and $P_{\mathrm{ref}}^{\mathrm{wfc}}=15 \mathrm{MW}$ (Scenario $3)$. The simulation time is $300 \mathrm{~s}$.

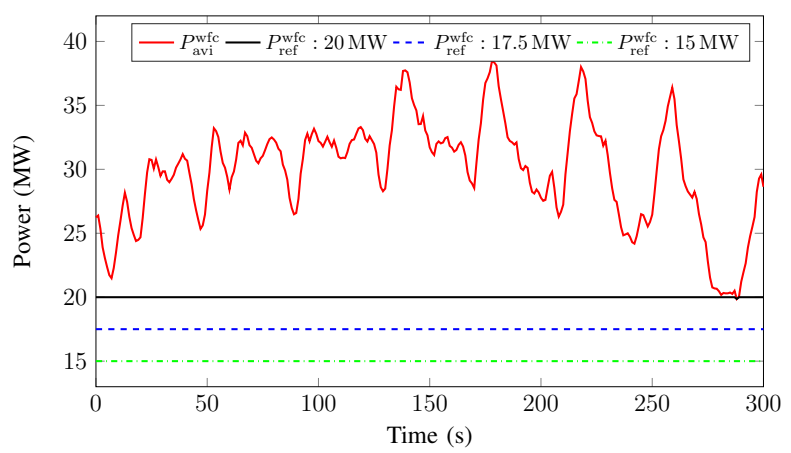

Fig. 9. $P_{\mathrm{avi}}^{\mathrm{wfc}}$ and $P_{\mathrm{ref}}^{\mathrm{wfc}}$ under the low wind condition.

1) Power Reference Tracking: To evaluate the tracking performance, the Root Mean Square Errors (RMSEs) for both algorithms are calculated and listed in Table I. Both values are identical and quite small, which implies the same tracking performances of both algorithms.

TABLE I

RMSE COMPARISON UNDER LOW WIND CONDITION

\begin{tabular}{c|c|c}
\hline \hline Scenario & CON & OPT \\
\hline \hline Scenario 1 & $0.012 \mathrm{MW}$ & $0.012 \mathrm{MW}$ \\
Scenario 2 & $0.003 \mathrm{MW}$ & $0.003 \mathrm{MW}$ \\
Scenario 3 & $0.003 \mathrm{MW}$ & $0.003 \mathrm{MW}$ \\
\hline
\end{tabular}

2) Fatigue Loads Reduction: Firstly, more details of the simulation result for Scenario 2 are shown. A representative WT (WT08) is used as an example. The variations of $T_{\mathrm{s}}$ with the two algorithms are shown in Fig. 10(a). Compared with the CON, the variation of the shaft torque $\Delta T_{\mathrm{s}}$ is reduced with the OPT. According to the cumulative rainflow cycles in Fig. 10(b), less cycles are found for the OPT, which implies less fatigue loads experienced by the WT.

The variations of $M_{\mathrm{t}}$ with the two algorithms are shown in Fig. 11(a). Compared with the CON, the variation of $\Delta M_{\mathrm{t}}$ is reduced with the OPT. According to the cumulative rainflow cycles in Fig. 11(b), less cycles are found in the large tower bending moment for the OPT, which implies less fatigue loads experienced by the WT.

The calculated DELs of $T_{\mathrm{s}}$ and $M_{\mathrm{t}}$ for all WTs are listed in Table II and III, respectively. As shown in Table II, with the OPT, most DELs are reduced. From the whole wind farm point of view, the total DEL of $T_{\mathrm{s}}$ is reduced by $2.11 \%$. Similarly, (a)

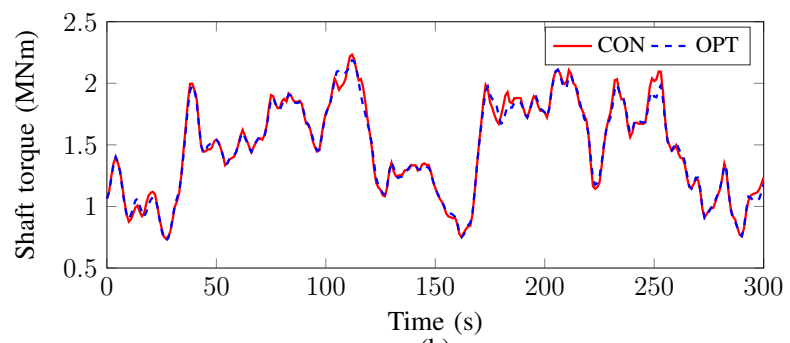

(b)

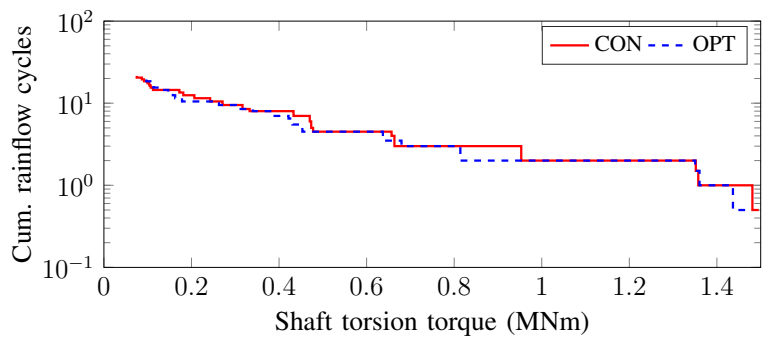

Fig. 10. $T_{\mathrm{S}}$ of WT08 for Scenario 2.

(a)

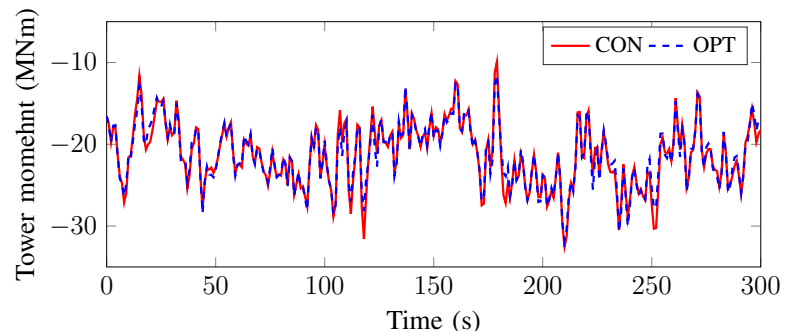

(b)

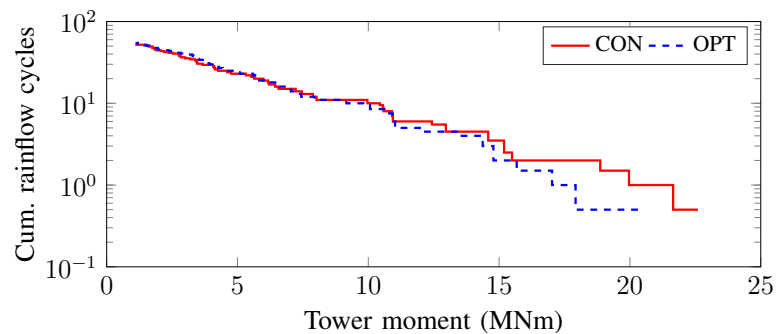

Fig. 11. $M_{\mathrm{t}}$ of WT08 for Scenario 2 .

as shown in Table III, most DELs are reduced. The reduction of the total DEL of $M_{\mathrm{t}}$ is $11.36 \%$.

The simulation results of Scenario 1-3 are summarized in Table IV. As mentioned in Section VII-A, $\Delta F_{\mathrm{t}}$ is more sensitive under the load wind condition. Therefore, for the DELs of $M_{\mathrm{t}}$, the reductions of the OPT are significant, which range from $11.36 \%$ to $13.32 \%$. Comparably, the changes of the DELs of $T_{\mathrm{s}}$ for the OPT are limited, which range from $0.73 \%$ to $-2.11 \%$.

\section{Operation under High Wind Condition}

The average wind speeds of all WTs $\left(v_{\mathrm{avr}}\right)$ in the wind farm are listed in Table VI, which range from $12.85 \mathrm{~m} / \mathrm{s}$ to $13.97 \mathrm{~m} / \mathrm{s}$. As shown in Fig. 12, three scenarios are defined according to different $P_{\mathrm{ref}}^{\mathrm{wfc}}: P_{\mathrm{ref}}^{\mathrm{wfc}}=45 \mathrm{MW}$ (Scenario 4), 
TABLE II

DELS OF WTS $\left(T_{\mathrm{S}}\right)$ FOR SCENARIO 2

\begin{tabular}{c|c|c|c|c}
\hline \hline No. & $\begin{array}{c}v_{\text {avr }} \\
\text { in m/s }\end{array}$ & $\begin{array}{c}\text { DEL (CON) } \\
\text { in MNm }\end{array}$ & $\begin{array}{c}\text { DEL (OPT) } \\
\text { in MNm }\end{array}$ & Percentage \\
\hline \hline WT01 & 8.85 & 1.88 & 1.86 & $-1.06 \%$ \\
WT02 & 9.09 & 2.10 & 2.07 & $-1.43 \%$ \\
WT03 & 9.46 & 1.75 & 1.64 & $-6.29 \%$ \\
WT04 & 9.10 & 1.86 & 1.74 & $-6.45 \%$ \\
WT05 & 9.75 & 1.47 & 1.51 & $2.72 \%$ \\
WT06 & 9.09 & 1.59 & 1.57 & $-1.26 \%$ \\
WT07 & 9.50 & 1.93 & 1.88 & $-2.59 \%$ \\
WT08 & 9.97 & 1.54 & 1.51 & $-1.95 \%$ \\
WT09 & 9.24 & 1.95 & 1.91 & $-2.05 \%$ \\
WT10 & 9.45 & 2.39 & 2.38 & $-0.42 \%$ \\
\hline \multicolumn{2}{c|}{ Summary } & 18.46 & 18.07 & $-2.11 \%$ \\
\hline
\end{tabular}

TABLE III

DELS OF WTS $\left(M_{\mathrm{t}}\right)$ FOR SCENARIO 2

\begin{tabular}{c|c|c|c|c}
\hline \hline No. & $\begin{array}{c}v_{\text {avr }} \\
\text { in m/s }\end{array}$ & $\begin{array}{c}\text { DEL (CON) } \\
\text { in MNm }\end{array}$ & $\begin{array}{c}\text { DEL (OPT) } \\
\text { in MNm }\end{array}$ & Percentage \\
\hline \hline WT01 & 8.85 & 26.70 & 26.00 & $-2.62 \%$ \\
WT02 & 9.09 & 31.72 & 25.15 & $-23.87 \%$ \\
WT03 & 9.46 & 27.77 & 21.19 & $-23.69 \%$ \\
WT04 & 9.10 & 34.94 & 27.95 & $-20.01 \%$ \\
WT05 & 9.75 & 19.35 & 20.16 & $4.19 \%$ \\
WT06 & 9.09 & 18.86 & 19.29 & $-2.28 \%$ \\
WT07 & 9.50 & 20.42 & 21.64 & $5.97 \%$ \\
WT08 & 9.97 & 22.82 & 20.02 & $-12.27 \%$ \\
WT09 & 9.24 & 33.41 & 30.32 & $-9.25 \%$ \\
WT10 & 9.45 & 33.40 & 28.06 & $-15.99 \%$ \\
\hline \multicolumn{6}{c}{ Summary } & 269.39 & 238.78 & $-11.36 \%$ \\
\hline
\end{tabular}

TABLE IV

COMPARISON OF DELS FOR DIFFERENT SCENARIOS

\begin{tabular}{c|c|c|c|c}
\hline \hline Scenario & Type & $\begin{array}{c}\text { DEL (CON) } \\
\text { in MNm }\end{array}$ & $\begin{array}{c}\text { DEL (OPT) } \\
\text { in MNm }\end{array}$ & Percentage \\
\hline \hline \multirow{2}{*}{ Scenario 1 } & $T_{\mathrm{s}}$ & 21.94 & 22.20 & $0.73 \%$ \\
& $M_{\mathrm{t}}$ & 404.16 & 350.34 & $-13.32 \%$ \\
\hline \multirow{2}{*}{ Scenario 2 } & $T_{\mathrm{s}}$ & 18.46 & 18.07 & $-2.11 \%$ \\
& $M_{\mathrm{t}}$ & 269.39 & 238.78 & $-11.36 \%$ \\
\hline \multirow{2}{*}{ Scenario 3 } & $T_{\mathrm{s}}$ & 14.86 & 14.82 & $-0.27 \%$ \\
& $M_{\mathrm{t}}$ & 213.19 & 187.63 & $-11.99 \%$ \\
\hline
\end{tabular}

$P_{\text {ref }}^{\mathrm{wfc}}=42.5 \mathrm{MW}$ (Scenario 5), and $P_{\mathrm{ref}}^{\mathrm{wfc}}=40 \mathrm{MW}$ (Scenario 6). The simulation time is $300 \mathrm{~s}$.

1) Power Reference Tracking: To evaluate the tracking performance, the RMSEs for both algorithms are calculated and listed in Table V. Both values are quite small, which shows good tracking performances of both algorithms. Most values of the OPT are the same as these of the CON.

2) Fatigue Loads Reduction: Firstly, more details of the simulation result for Scenario 5 are shown. A representative WT (WT05) is used as an example. The variations of $T_{\mathrm{s}}$ with the two algorithms are shown in Fig. 13(a). Compared

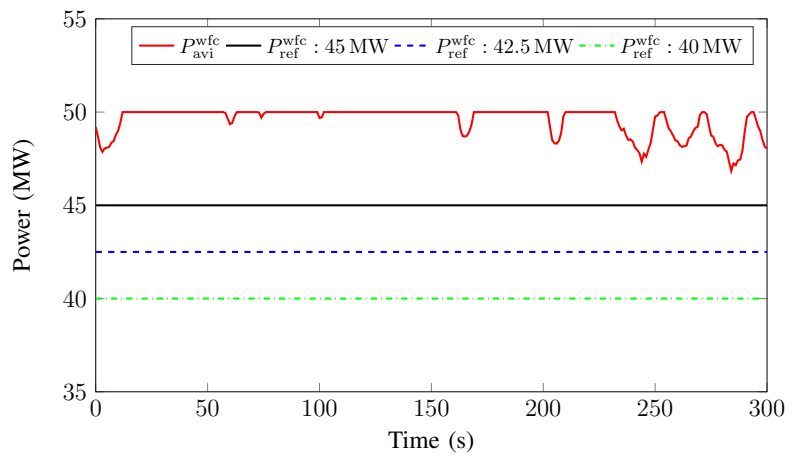

Fig. 12. $P_{\mathrm{avi}}^{\mathrm{wfc}}$ and $P_{\mathrm{ref}}^{\mathrm{wfc}}$ under the high wind condition.

TABLE V

RMSE COMPARISON UNDER HIGH WIND CONDITION

\begin{tabular}{c|c|c}
\hline \hline Scenario & CON & OPT \\
\hline \hline Scenario 4 & $0.010 \mathrm{MW}$ & $0.009 \mathrm{MW}$ \\
Scenario 5 & $0.009 \mathrm{MW}$ & $0.009 \mathrm{MW}$ \\
Scenario 6 & $0.008 \mathrm{MW}$ & $0.008 \mathrm{MW}$ \\
\hline
\end{tabular}

with the CON, the variation of $\Delta T_{\mathrm{s}}$ is reduced with the OPT. According to the cumulative rainflow cycles in Fig. 13(b), less cycles are found for the OPT, which implies less fatigue loads experienced by the WT.

(a)

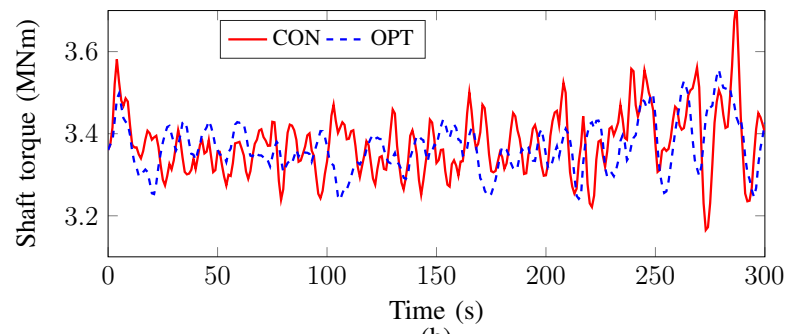

(b)

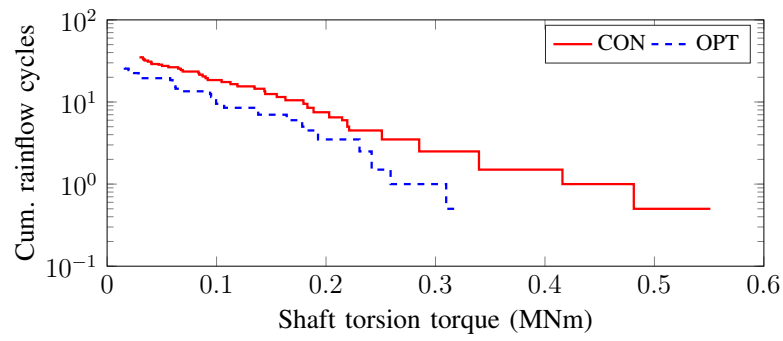

Fig. 13. $T_{\mathrm{s}}$ of WT05 for Scenario 5.

The variations of $M_{\mathrm{t}}$ with the two algorithms are shown in Fig. 14(a). Compared with the CON, the variation of $\Delta M_{\mathrm{t}}$ is reduced with the OPT. According to the cumulative rainflow cycles in Fig. 14(b), less cycles are found in the large tower moment for the OPT, which implies less fatigue loads experienced by the WT.

The calculated DELs of $T_{\mathrm{s}}$ and $M_{\mathrm{t}}$ for all WTs are listed in Table VI and VII, respectively. As shown in Table VI, compared with the CON, all the DELs are reduced with the OPT. The reduction values are from $0.00 \%$ to $39.62 \%$. From 
(a)

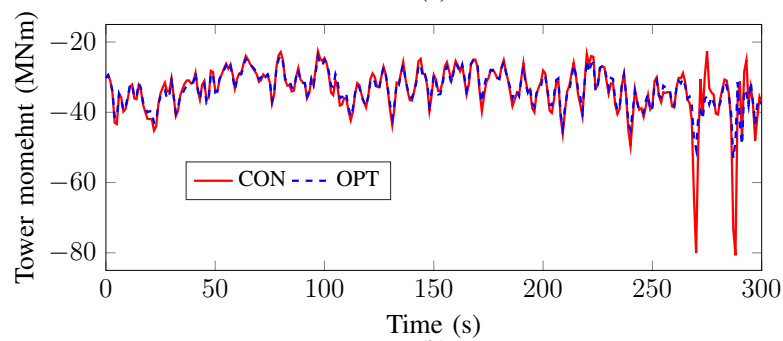

(b)

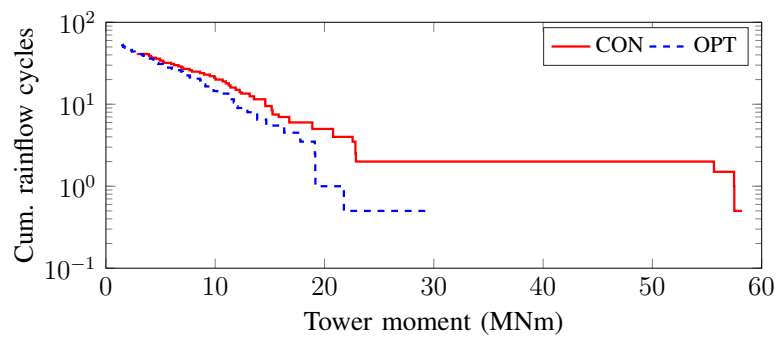

Fig. 14. $M_{\mathrm{t}}$ of WT05 for Scenario 5 .

the whole wind farm point of view, the total DEL of $T_{\mathrm{S}}$ is reduced by $14.88 \%$. Similarly, as shown in Table VII, most DELs are reduced. The reduction of the total DEL of $M_{\mathrm{t}}$ is $21.19 \%$

TABLE VI

DELS OF WTS $\left(T_{\mathrm{S}}\right)$ FOR SCENARIO 5

\begin{tabular}{c|c|c|c|c}
\hline \hline No. & $\begin{array}{c}v_{\text {avr }} \\
\text { in m/s }\end{array}$ & $\begin{array}{c}\text { DEL (CON) } \\
\text { in MNm }\end{array}$ & $\begin{array}{c}\text { DEL (OPT) } \\
\text { in MNm }\end{array}$ & Percentage \\
\hline \hline WT01 & 12.85 & 1.44 & 1.21 & $-15.97 \%$ \\
WT02 & 13.09 & 1.37 & 1.19 & $-13.14 \%$ \\
WT03 & 13.46 & 1.35 & 1.11 & $-17.78 \%$ \\
WT04 & 13.10 & 1.52 & 1.43 & $-5.92 \%$ \\
WT05 & 13.75 & 0.53 & 0.32 & $-39.62 \%$ \\
WT06 & 13.09 & 0.61 & 0.45 & $-26.23 \%$ \\
WT07 & 13.50 & 0.36 & 0.32 & $-11.11 \%$ \\
WT08 & 13.97 & 0.57 & 0.57 & $-0.00 \%$ \\
WT09 & 13.24 & 0.45 & 0.36 & $-20.00 \%$ \\
WT10 & 13.45 & 1.21 & 1.05 & $-13.22 \%$ \\
\hline \multicolumn{2}{c|}{ Summary } & 9.41 & 8.01 & $-14.88 \%$ \\
\hline
\end{tabular}

The simulation results of Scenario 4-6 are summarized in Table VIII. Compared with the CON, the DELs of both $T_{\mathrm{s}}$ and $M_{\mathrm{t}}$ with the OPT are reduced significantly. Specifically, for $T_{\mathrm{s}}$, the reduction values range from $10.97 \%$ to $20.77 \%$. For $M_{\mathrm{t}}$, the reduction values range from $16.24 \%$ to $25.37 \%$.

3) Discussion: The relation between the DEL value and $P_{\mathrm{ref}}^{\mathrm{wfc}}$ is analyzed. By taking the DEL value of $P_{\mathrm{ref}}^{\mathrm{wfc}}=$ $45 \mathrm{MW}$ as the reference, different $P_{\text {ref }}^{\mathrm{wfc}}$ are sampled and the corresponding DELs in p.u. are calculated. According to these points, the fitted curve can be derived based on the nonlinear least square algorithm, as shown in Fig. 15. It can be observed that the DEL can be reduced by decreasing the wind power generation. However, with smaller $P_{\text {ref }}^{\mathrm{wfc}}$, the reduction sensitivity becomes smaller. For the wind farm operator, the damage to the WTs might be also considered to decide $P_{\text {ref }}^{\text {wfc }}$.
TABLE VII

DELS OF WTS $\left(M_{\mathrm{t}}\right)$ FOR SCENARIO 5

\begin{tabular}{c|c|c|c|c}
\hline \hline No. & $\begin{array}{c}v_{\text {avr }} \\
\text { in m/s }\end{array}$ & $\begin{array}{c}\text { DEL (CON) } \\
\text { in MNm }\end{array}$ & $\begin{array}{c}\text { DEL (OPT) } \\
\text { in MNm }\end{array}$ & Percentage \\
\hline \hline WT01 & 12.85 & 57.75 & 45.07 & $-21.96 \%$ \\
WT02 & 13.09 & 48.66 & 46.41 & $-4.62 \%$ \\
WT03 & 13.46 & 46.16 & 44.13 & $-4.40 \%$ \\
WT04 & 13.10 & 41.88 & 42.09 & $0.50 \%$ \\
WT05 & 13.75 & 61.38 & 27.65 & $-54.95 \%$ \\
WT06 & 13.09 & 49.60 & 26.56 & $-46.45 \%$ \\
WT07 & 13.50 & 27.31 & 24.39 & $-10.69 \%$ \\
WT08 & 13.97 & 51.74 & 48.97 & $-5.35 \%$ \\
WT09 & 13.24 & 32.40 & 28.54 & $-11.91 \%$ \\
WT10 & 13.45 & 54.32 & 37.54 & $-30.89 \%$ \\
\hline \multicolumn{2}{c|}{ Summary } & 471.20 & 371.35 & $-21.19 \%$ \\
\hline
\end{tabular}

TABLE VIII

COMPARISON OF DELS FOR DIFFERENT SCENARIOS

\begin{tabular}{c|c|c|c|c}
\hline \hline Scenario & Type & $\begin{array}{c}\text { DEL (CON) } \\
\text { in MNm }\end{array}$ & $\begin{array}{c}\text { DEL (OPT) } \\
\text { in MNm }\end{array}$ & Percentage \\
\hline \hline \multirow{2}{*}{ Scenario 4 } & $T_{\mathrm{S}}$ & 10.67 & 9.5 & $-10.97 \%$ \\
& $M_{\mathrm{t}}$ & 513.57 & 430.16 & $-16.24 \%$ \\
\hline \multirow{2}{*}{ Scenario 5 } & $T_{\mathrm{S}}$ & 9.41 & 8.01 & $-14.88 \%$ \\
& $M_{\mathrm{t}}$ & 471.20 & 371.35 & $-21.19 \%$ \\
\hline \multirow{2}{*}{ Scenario 6 } & $T_{\mathrm{S}}$ & 8.86 & 7.02 & $-20.77 \%$ \\
& $M_{\mathrm{t}}$ & 431.44 & 321.99 & $-25.37 \%$ \\
\hline
\end{tabular}

Sometimes, it might be more beneficial to sacrifice $P_{\mathrm{ref}}^{\mathrm{wfc}}$ to reduce the damages to the WTs. How to evaluate the economical benefit of the DEL reduction and strike a balance between the DEL reduction and wind power generation will be studied in the future work.

(a)
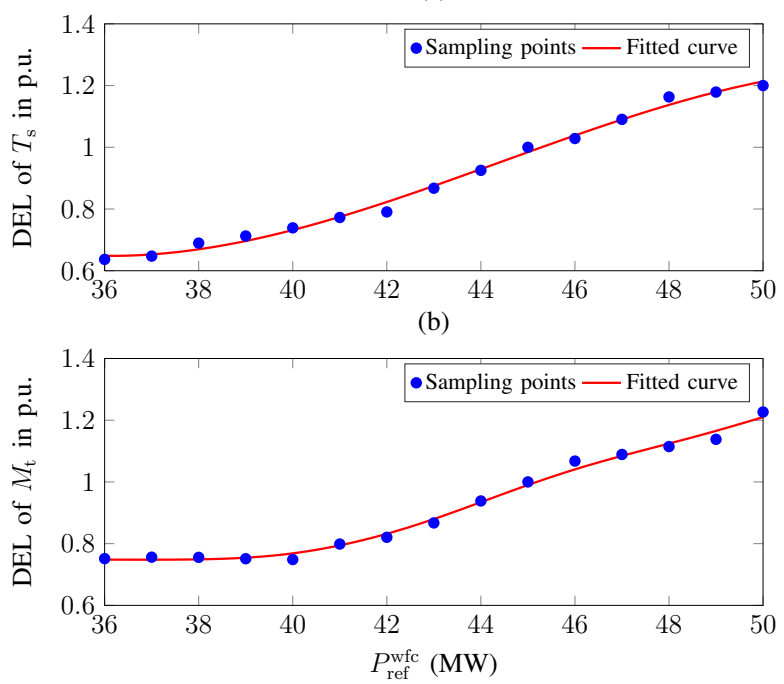

Fig. 15. Relation between DEL and $P_{\text {ref }}^{\text {wfc }}$. 


\section{CONCLUSION}

In this paper, an optimal dispatch algorithm based on fatigue load sensitivity is developed for the active power control of wind farms. The load sensitivity and feasible range of the power reference for individual WTs are used to formulate the optimal dispatch problem, which are calculated locally. With this configuration, most computation tasks are distributed to the local WT controllers. The complicated wind farm model is not required and the computation burden of the wind farm controller is largely reduced. Moreover, the explicit analytical equations of the load sensitivity are derived to improve the computation efficiency of the local controller. The proposed dispatch algorithm is suitable for real-time control of largescale wind farms. Case studies show the developed algorithm can achieve good performances of power tracking. Moreover, the fatigue loads experienced by WTs are largely alleviated.

\section{APPENDIX}

The parameters of $5 \mathrm{MW}$ wind turbine model are listed in Table IX.

TABLE IX

PARAMETER OF 5 MW WIND TURBINE MODEL

\begin{tabular}{l|l|l}
\hline Symbol & Description & value \\
\hline \hline$\eta$ & Multiplier ratio & 97 \\
$R$ & Rotor blade length $(\mathrm{m})$ & 63 \\
$H$ & Height of the rotor center $(\mathrm{m})$ & 87.6 \\
$J_{\mathrm{r}}$ & Rotor inertia $\left(\mathrm{kg} \cdot \mathrm{m}^{2}\right)$ & $3.544 \cdot 10^{7}$ \\
$J_{\mathrm{g}}$ & Generator inertia $\left(\mathrm{kg} \cdot \mathrm{m}^{2}\right)$ & 534.116 \\
$k_{\mathrm{sh}}$ & Drive train spring constant $(\mathrm{N} \cdot \mathrm{m} / \mathrm{rad})$ & $8.676 \cdot 10^{8}$ \\
$c_{\mathrm{sh}}$ & Drive train damping constant & $6.215 \cdot 10^{6}$ \\
& (N·m·s/rad) & \\
$M_{\mathrm{tow}}$ & Integrated tower mass $(\mathrm{kg})$ & $3.475 \cdot 10^{5}$ \\
$\omega_{\mathrm{g} \_ \text {rated }}$ & Rated generator speed $(\mathrm{rad} / \mathrm{s})$ & 122.91 \\
$\omega_{\mathrm{r} \_ \text {rated }}$ & Rated rotor speed $(\mathrm{rad} / \mathrm{s})$ & 1.26 \\
$\theta_{\mathrm{max}}$ & Max. pitch angle $(\mathrm{deg})$ & 90 \\
$\theta_{\mathrm{min}}$ & Min. pitch angle $(\mathrm{deg})$ & 0 \\
$K_{\mathrm{p}}$ & Proportional gain of the pitch control & 0.2143 \\
$K_{\mathrm{i}}$ & Integral gain of the pitch control & 0.0918 \\
$K_{0}$ & Gain scheduling coefficient & 1 \\
$K_{\theta}$ & Gain scheduling coefficient & 2.1323 \\
\hline
\end{tabular}

\section{REFERENCES}

[1] I. Pineda et al., "Wind in power-2015 European statistics," EWEA, Brussels, Belgium, Tech. Rep., Feb. 2016.

[2] M. Kayikci and J. V. Milanović, "Dynamic contribution of DFIG-based wind plants to system frequency disturbances," IEEE Trans. Power Syst., vol. 24, no. 2, pp. 859-867, May 2009.

[3] C. Wan, Z. Xu, P. Pinson et al., "Probabilistic forecasting of wind power generation using extreme learning machine," IEEE Trans. Power Syst., vol. 29, no. 3, pp. 1033-1044, May 2014.

[4] C. Wan, Z. Xu, P. Pinson et al., "Optimal prediction intervals of wind power generation," IEEE Trans. Power Syst., vol. 29, no. 3, pp. 11661174, May 2014.

[5] M. Tsili and S. Papathanassiou, "A review of grid code technical requirements for wind farms," IET Renew. Power Gen., vol. 3, no. 3, pp. 308-332, Sep. 2009.
[6] P. E. Sørensen, A. D. Hansen, K. Thomsen et al., "Operation and control of large wind turbines and wind farms," Risø Nat. Lab., Roskilde, Denmark, Tech. Rep. Risø-R-1532, Sep. 2005.

[7] P. E. Sørensen, A. D. Hansen, F. Iov et al., "Wind farm models and control strategies," Ris $\varnothing$ Nat. Lab., Roskilde, Tech. Rep. Ris $\varnothing-R-1464$ Aug. 2005.

[8] Z. Lubosny and J. W. Bialek, "Supervisory control of a wind farm," IEEE Trans. Power Syst., vol. 22, no. 3, pp. 985-994, Jul. 2007.

[9] N. Khezami, N. B. Braiek, and X. Guillaud, "Wind turbine power tracking using an improved multimodel quadratic approach," ISA Trans., vol. 49, no. 3, pp. 326-334, Jul. 2010.

[10] H. Camblong, S. Nourdine, I. Vechiu, and G. Tapia, "Control of wind turbines for fatigue loads reduction and contribution to the grid primary frequency regulation," Energy, vol. 48, no. 1, pp. 284-291, Dec. 2012.

[11] J. B. Berglind, R. Wisniewski, and M. Soltani, "Fatigue load modeling and control for wind turbines based on hysteresis operators," in IEEE American Control Conf., Chicago, IL, 2015, pp. 3721-3727.

[12] V. Spudić, M. Jelavić, and M. Baotić, "Wind turbine power references in coordinated control of wind farms," Automatika-Journal for Control, Measurement, Electronics, Computing and Communications, vol. 52, no. 2, pp. 82-94, Jul. 2011.

[13] V. Spudić, M. Jelavic, M. Baotic et al., "Hierarchical wind farm control for power/load optimization," in The Science of making Torque from Wind (Torque2010), Heraklion, Greece, 2010.

[14] V. Spudić, "Coordinated optimal control of wind farm active power," Ph.D. dissertation, Dept. Control Comput. Eng., Univ. of Zagreb, Zagreb, Croatia, 2012.

[15] B. Biegel, D. Madjidian, V. Spudic et al., "Distributed low-complexity controller for wind power plant in derated operation," in IEEE Int. Conf. Control Applicat. (CCA), Hyderabad, India, 2013, pp. 146-151.

[16] H. Zhao, Q. Wu, Q. Guo et al., "Distributed model predictive control of a wind farm for optimal active power control- Part I: Clustering-based wind turbine model linearization," IEEE Trans. Sustain. Energy, vol. 6, no. 3, pp. 831-839, Jul. 2015.

[17] H. Zhao, Q. Wu, Q. Guo et al., "Distributed model predictive control of a wind farm for optimal active power control-Part II: Implementation with clustering-based piece-wise affine wind turbine model," IEEE Trans. Sustain. Energy, vol. 6, no. 3, pp. 840-849, Jul. 2015

[18] V. Spudić, C. Conte, M. Baotić et al., "Cooperative distributed model predictive control for wind farms," Optim. Control Appl. Methods, vol. 36, no. 3, pp. 333-352, May 2015.

[19] T. Knudsen, T. Bak, and M. Svenstrup, "Survey of wind farm controlpower and fatigue optimization," Wind Energy, vol. 18, no. 8, pp. 1333-1351, Aug. 2015.

[20] J. M. Jonkman, S. Butterfield et al., "Definition of a 5-MW reference wind turbine for offshore system development", Nat. Renew. Energy Lab., Denver, CO, Tech. Rep. NREL/TP-500-38060, Feb. 2009.

[21] J. D. Grunnet, M. Soltani, T. Knudsen et al., "Aeolus toolbox for dynamic wind farm model, simulation and control," in The European Wind Energy Conf. \& Exihib., Warsaw, Poland, Apr. 2010.

[22] M. Vašak, N. Hure, and N. Perić, "Identification of a discrete-time piecewise affine model of a pitch-controlled wind turbine," in Proc the 34th Int. Conv. MIPRO, Opatija, Crotia, May 2011, pp. 744-749.

[23] Handbook of wind power systems, Springer, Berlin, Germany, Jan. 2014.

[24] O. Anaya-Lara, N. Jenkins et al., Wind energy generation: modelling and control, Chichester, UK: John Wiley \& Sons Ltd, Jul. 2009.

[25] J. F. Manwell, J. G. McGowan et al., Wind energy explained: theory, design and application, 2nd ed., Chichester, UK: John Wiley \& Sons Ltd, Dec. 2009.

[26] T. K. Barlas and G. Van Kuik, "Review of state of the art in smart rotor control research for wind turbines," Prog. Aerosp. Sci., vol. 46, no. 1, pp. 1-27, Jan. 2010.

[27] M. N. Soltani, T. Knudsen, M. Svenstrup et al., "Estimation of rotor effective wind speed: A comparison," IEEE Trans. Control Syst. Technol., vol. 21, no. 4, pp. 1155-1167, Jun. 2013.

[28] T. Ackermann et al., Wind power in power systems, 2nd ed., Chichester, UK: John Wiley \& Sons Ltd, Jan. 2005.

[29] M. Baotić, "Optimal control of piecewise affine systemsa mutliparametric approach," Ph.D. dissertation, ETH Zürich, Zürich, Switzerland, Jul. 2005.

[30] I. Munteanu, A. Bratcu et al., Optimal control of wind energy systems: towards a global approach, 1st ed., London, UK: Springer, 2008.

[31] Wind energy handbook, 2nd ed., John Wiley \& Sons Ltd, Chichester, UK, May 2011.

[32] MCrunch user's guide for version 1.00, 1st ed., Nat. Renew. Energy Lab., Denver, CO, May 2008. 


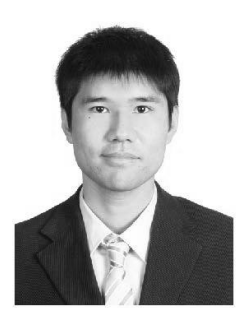

Haoran Zhao (S'12-M'15) received the B.E. degree in electrical engineering and automation from Shandong University, Jinan, China, in 2005, the M.E. degree in electrical engineering and automation from the Technical University of Berlin, Berlin, Germany, in 2009, and the Ph.D. degree in electrical engineering from the Technical University of Denmark, Kgs. Lyngby, Denmark, in 2015.

$\mathrm{He}$ is a Postdoc with the Center for Electric Technology, Technical University of Denmark. He worked as Electrical Engineer with State Grid Corporation of China (SGCC), Beijing, China, in 2005. From 2010 to 2011, he worked as Application Developer with DIgSILENT GmbH, Gomaringen, Germany. His research interests include modeling and integration study of wind power, control of energy storage system, and voltage stability analysis.

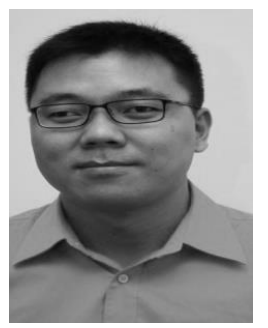

Qiuwei Wu (M'08-SM'15) obtained the B. Eng. and M. Eng. in Power System and Its Automation from Nanjing University of Science and Technology, Nanjing, China, in 2000 and 2003, respectively. $\mathrm{He}$ obtained the PhD degree in Power System Engineering from Nanyang Technological University, Singapore, in 2009.

He was a senior R\&D engineer with VESTAS Technology R\&D Singapore Pte Ltd from Mar. 2008 to Oct. 2009. He has been working at Department of Electrical Engineering, Technical University of Denmark (DTU) since Nov. 2009 (PostDoc Nov. 2009-Oct. 2010, Assistant Professor Nov. 2010-Aug. 2013, Associate Professor since Sept. 2013). He was a visiting scholar at Department of Industrial Engineering \& Operations Research (IEOR), University of California, Berkeley, from Feb. 2012 to May 2012 funded by Danish Agency for Science, Technology and Innovation (DASTI), Denmark. He has been a visiting professor named by Y. Xue, an Academician of Chinese Academy of Engineering, at Shandong University, China, since Nov. 2015.

His research interests are smart grids, wind power, electric vehicle, active distribution networks, electricity market, and smart energy systems. He is an Editor of IEEE Transactions on Smart Grid and IEEE Power Engineering Letters. He is also an Associate Editor of International Journal of Electrical Power and Energy Systems.

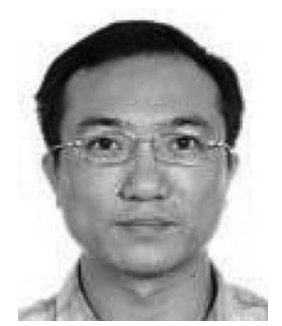

Shaojun Huang (S'13) obtained the B.Eng. from the Department of Electrical Engineering, Tsinghua University, Beijing, China, in 2001. He obtained the MSc from the Department of Energy Technology, Aalborg University, Denmark, in 2013.

Currently, he is a Postdoc in the Centre for Electric Power and Energy, Department of Electrical Engineering, Technical University of Denmark.

His research interests are congestion management for distribution networks with high penetration of distributed energy resources.

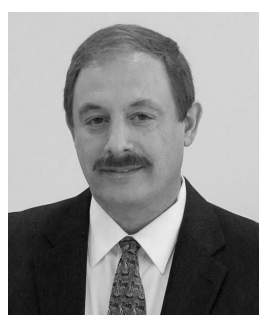

Mohammad Shahidehpour (F'01) is the Bodine Chair Professor with the Department of Electrical and Computer Engineering, the Director of the Robert W. Galvin Center for Electricity Innovation, and an Associate Director of WISER with the Illinois Institute of Technology, Chicago, IL, USA

He was a recipient of the IEEE PES Outstanding Power Engineering Educator Award, the IEEE PES Outstanding Engineer Award, and the Chicago Chapter. $\mathrm{He}$ is the Holder of Nourbakhshian Endowed Chair Professorship, University of Kashan, Iran, and Otto Monsted Professorship, Technical University of Denmark. He is a Research Professor with King Abdulaziz University, Saudi Arabia, the Sharif University of Technology, Iran, as well as several universities in China, including Tsinghua University, Xian Jiaotong University, Nanjing University, North China Electric Power University, and Hunan University. He is an IEEE PES Distinguished Lecturer, and served as the VP of Publications for the IEEE Power and Energy Society, the Editor-in-Chief of the IEEE TRANSACTIONS ON POWER SYSTEMS, and the Founding Editor-in-Chief of the IEEE TRANSACTIONS ON SMART GRID.

Dr. Shahidehpour is a member of the U.S. National Academy of Engineering.

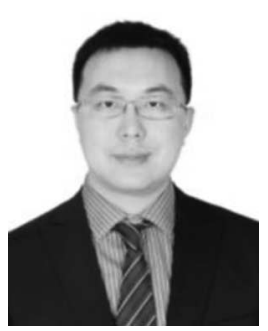

Qinglai Guo (M'09-SM'14) was born in Jilin, China, on March 6, 1979. He received the B.S. and $\mathrm{Ph} . \mathrm{D}$. degrees in electrical engineering from Tsinghua University, Beijing, China, in 2000 and 2005 , respectively.

$\mathrm{He}$ is currently an Associate Professor with Tsinghua University. His research interests include smart grids, cyber-physical systems, and electrical power control center applications.

Prof. Guo is a member of CIGRE C2.13 Task Force on Voltage/Var support in System Operations.

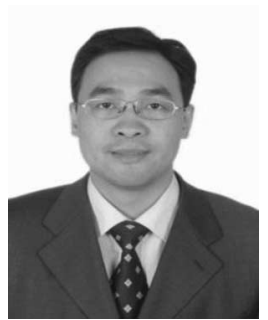

Hongbin Sun (M'00-SM'12) received double B.S. degrees in electrical engineering and physics from Tsinghua University, Beijing, China, in 1992, and the Ph.D. degree in electrical engineering from Tsinghua University in 1997.

$\mathrm{He}$ is now the Changjiang Scholar of Education Ministry of China, full Professor of Electrical Engineering with Tsinghua University and Assistant Director with the State Key Laboratory of Power Systems, China. From 2007 to 2008, he was a Visiting Professor with the School of EECS at Washington State University, Pullman, WA, USA. He has authored more than 300 academic papers. His research interests include smart grids, renewable generation integration, and electrical power control center applications.

$\mathrm{He}$ is a Fellow of IET. He is a member of IEEE PES CAMS Cascading Failure Task Force and CIGRE C2.13 Task Force on Voltage/Var support in System Operations. He was the recipient of the China National Technology Innovation Award in 2008, the National Distinguished Teacher Award in China in 2009, and the National Science Fund for Distinguished Young Scholars of China in 2010. 